\title{
2 In Situ Radiation Damage Studies of Optoelectronics in 3 the ATLAS SemiConductor Tracker
}

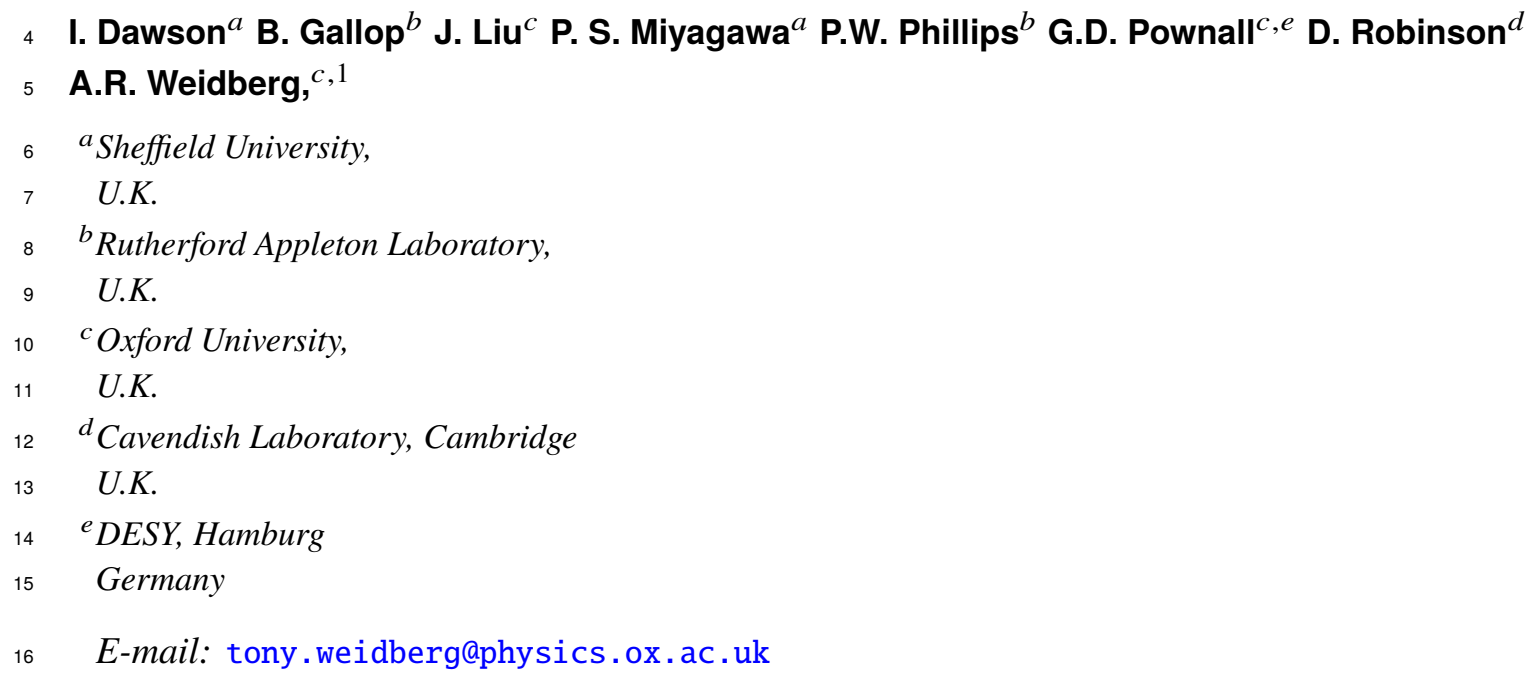

\begin{abstract}
This paper presents results on in situ measurements of radiation damage for the ondetector optoelectronics for the ATLAS SemiConductor Tracker. The results come from protonproton collisions in LHC during operation in 2016, 2017 and 2018. The results for both $p-i-n$ diodes and VCSELs are given and compared to expectations from beam tests of devices from the same wafer, before the start of LHC operation. The VCSELs show evidence for some radiation damage as expected from earlier studies. The magnitude of the damage is compared with simple extrapolations from test beam data. The $p-i-n$ diodes do show significant radiation damage as expected from test beam studies.
\end{abstract}

KeYwords: Radiation-hard detectors, Photon detectors for IR photons (solid-state), lasers

${ }^{1}$ Corresponding author. 


\section{Contents}

1 Introduction $\quad 1$

2 Radiation Damage Studies Prior to LHC Operation $\quad 2$

2.1 VCSELs 2

$2.2 p-i$ - $n$ Diodes 3

3 Predicted Particle Fluences $\quad 3$

4 In situ measurements at the LHC $\quad 5$

4.1 VCSELs $\quad 5$

$4.2 p-i-n$ Diodes 11

4.2.1 Photo-currents 11

$\begin{array}{lll}\text { 4.2.2 Depletion Voltage } & 18\end{array}$

$\begin{array}{lll}4.3 & \text { Discussion of Results } & 21\end{array}$

5 Conclusion $\quad 26$

$\begin{array}{lr}\text { A Stability study } & 26\end{array}$

\section{Introduction}

Optical links are used for the readout and control of the SemiConductor Tracker (SCT) [1],[2],[3]. The data links are based on AlGaAs Vertical Cavity Surface Emitting Lasers (VCSELs) ${ }^{1}$ on the detector and silicon $p-i$ - $n$ diodes in the counting room. The Timing, Trigger and Control (TTC) links use AlGaAs VCSELs in the counting room and silicon $p-i-n$ diodes $^{2}$ on the detector.

This paper compares the results of radiation damage studies for VCSELs and $p-i$ - $n$ diodes performed before the start of ATLAS operation with the observed radiation damage during protonproton collisions in the LHC at a centre of mass energy of $13 \mathrm{TeV}$. The integrated luminosity delivered to ATLAS [4] for the period 2016 to 2018 is given in Table 1. The results of the radiation damage studies performed before the start of ATLAS operation are summarised in section 2. Radiation damage studies from these test beams have been used to predict the radiation damage expected during ATLAS operation assuming that the damage scales with Non-Ionising Energy Loss (NIEL). While the NIEL scaling hypothesis is known to work well for silicon [5], there are much larger uncertainties in the use of NIEL scaling for GaAs devices [6]. In addition the fluxes were

${ }^{1}$ In VCSELs a very high reflectivity is achieved using alternating layers of GaAs and GaAlAs to make a Distributed Bragg Reflector (DBR) structure. The light is emitted from GaAs multi-quantum wells sandwiched between the top and bottom DBR. The on-detector VCSELs were manufactured by Truelight, Taiwan, TSD-8A12.

${ }^{2}$ AEP 10, manufactured by Centronic, Croydon, UK. 
Table 1: Integrated luminosity delivered to ATLAS in the period 2016-2018.

\begin{tabular}{|c|l|}
\hline Year & Integrated Luminosity $\left(\mathrm{fb}^{-1}\right)$ \\
\hline 2016 & 38.5 \\
2017 & 50.2 \\
2018 & 65.2 \\
\hline
\end{tabular}

vastly higher in the test beam studies compared to those obtained in ATLAS operation. Therefore it is interesting to compare the measured in situ results with the extrapolations from previous test beam studies. The expected fluences during SCT operation are summarised in section 3. The methods used for in situ monitoring of the optical links in the SCT are described in section 4. The radiation damage determined in situ for the VCSELs ( $p-i-n$ diodes) is described in section 4.1 (4.2) and compared to the previous data in section 4.3. Some conclusions are given in section 5.

\section{Radiation Damage Studies Prior to LHC Operation}

\subsection{VCSELS}

VCSELs are sensitive to bulk damage from high energy particles. These create defects in the GaAs lattice which can lead to non-radiative centres thus reducing the VCSEL slope efficiency ${ }^{3}$ and increase the laser threshold [7]. A sample of 20 VCSELs from the wafer used for the SCT was irradiated to a fluence of $2 \times 10^{14} \mathrm{p} \mathrm{cm}^{-2}$ using protons with a kinetic energy of $30 \mathrm{MeV}$, and a sample of 8 VCSELs from the same wafer were irradiated using protons with an energy of $24 \mathrm{GeV}$ with a fluence of $1.09 \times 10^{14} \mathrm{p} \mathrm{cm}^{-2}$ [8]. Studies of radiation damage for similar VCSELs were performed using neutrons with a kinetic energy around $1 \mathrm{MeV}$ and a fluence of $5 \times 10^{14} \mathrm{n} \mathrm{cm}^{-2}$ [7].

Assuming that the damage in GaAs scales with Non-Ionising Energy Loss (NIEL) [6], the damage at these fluences can be compared to each other and to that expected during SCT operation at the LHC [8]. The fluence obtained with the $30 \mathrm{MeV}$ protons is estimated to be equivalent to that expected for the worst case in the SCT after an integrated luminosity of $\sim 1000 \mathrm{fb}^{-1}$. This is a factor of $\sim 3$ larger than what is now expected during the operational lifetime of the SCT. Significant increases in the laser threshold and decreases in slope efficiency were observed. At the end of the irradiation, the slope efficiency decreased to a value of $(73 \pm 4) \%$ compared to pre-irradiation and the threshold increased by $3.0 \pm 0.4 \mathrm{~mA}$. However after the exposure, the devices showed very strong beneficial annealing. The beneficial annealing depends strongly on the VCSEL bias current. Although there are no theoretical models that can predict this annealing, empirical models have been used to extrapolate short exposures and annealing periods to the much longer exposure periods expected during High Luminosity LHC (HL-LHC) operation [9]. After one week of operation at a VCSEL bias current of $10 \mathrm{~mA}$, the slope efficiency increased to $(88 \pm 4) \%$ of the pre-irradiation value. In addition, the threshold increase with radiation decreased to $0.94 \pm 0.11 \mathrm{~mA}$. After a further week of operation at $20 \mathrm{~mA}$ drive current, complete annealing of the slope efficiency was observed and the change in threshold was reduced to a value of $0.42 \pm 0.13 \mathrm{~mA}$, which is much

${ }^{3}$ The slope efficiency is the gradient of a linear fit to the optical power versus laser drive current above the laser threshold. 

regions of similar fluences as defined in Table 2.

Table 2: Definition of three regions of End-caps. The regions are defined so as to correspond to

similar fluences.

\begin{tabular}{|l|l|}
\hline Region & Disks and rings included \\
\hline 1 & Disks 1-6 outer regions and disk 1 middle region \\
2 & Disks 7-8 outer regions and disks 2-6 middle regions \\
3 & Disk 9 outer region, disks 7-8 middle regions and disks 2-6 inner regions \\
\hline
\end{tabular}

less than than the threshold before irradiation of $3.28 \pm 0.09 \mathrm{~mA}$. Compatible results were obtained with the irradiation using $24 \mathrm{GeV} / \mathrm{c}$ protons [8]. As expected for VCSELs there was no evidence of radiation damage from ionising radiation using a ${ }^{60} \mathrm{Co}$ source.

\section{$2.2 p-i-n$ Diodes}

Radiation damage studies of the $p-i-n$ diodes used in the SCT were performed using neutrons and proton beams of different energies [10],[11]. The resulting fluences were converted to an equivalent fluence of $1 \mathrm{MeV}$ neutrons using the NIEL hypothesis [5]. The results showed that there was a rapid decrease in responsivity ${ }^{4}$ up to a fluence of $\sim 2.5 \times 10^{13} 1 \mathrm{MeVn}$ eq $/ \mathrm{cm}^{2}$. However beyond this fluence very little further decrease in responsivity was observed. For a fluence of $3 \times 10^{14} 1 \mathrm{MeVn}_{\mathrm{eq}} / \mathrm{cm}^{2}$, the responsivity decreased by $29 \%$ relative to the values before irradiation.

Scans of the photo-current versus bias voltage were performed before irradiation and at three different fluences to determine changes in depletion voltages. The depletion voltage increased to $\sim 1 \mathrm{~V}$ after a fluence of $\sim 1.5 \times 10^{14} 1 \mathrm{MeVn}_{\mathrm{eq}} / \mathrm{cm}^{2}$. This is a factor of $\sim 2$ larger than the fluence expected for the inner layer of the SCT after an integrated luminosity of $\mathcal{L}=350 \mathrm{fb}^{-1}$. Therefore given the luminosity that has already been acquired, we do not expect any significant change in depletion voltage.

\section{Predicted Particle Fluences}

The FLUKA particle transport code [12],[13] was used to perform radiation background predictions in the innermost part of the ATLAS detector, including the SCT. Simulations are performed using the same criteria as described in refs. [1],[14], which also give definitions and explanations of the radiation quantities used. Hadronic and electromagnetic interactions and cascades are typically transported from $\mathrm{TeV}$ energies down to $\mathrm{keV}$ (or thermal energies in the case of neutrons). The Pythia8 event generator [15] with ATLAS A3 tune [16] was used to simulate the inelastic protonproton ( $p p$ ) collisions. A schematic view of the ATLAS Inner Detector is shown in fig. 1.

The analysis of radiation damage is grouped into regions which receive similar fluences. In the barrel region of the detector, the fluence varies rapidly with the radial distance to the beam line $(r)$ but only slowly with the distance along the beam direction $(z)$. In the End-cap region, the fluence varies rapidly with both $r$ and $z$. Therefore for the barrel region the data were split into sets corresponding to the four barrels (see fig. 1). For the End-cap regions, the data were split into three

${ }^{4}$ The responsivity for a photo-diode is defined to be the photo-current divided by the incident optical power. 


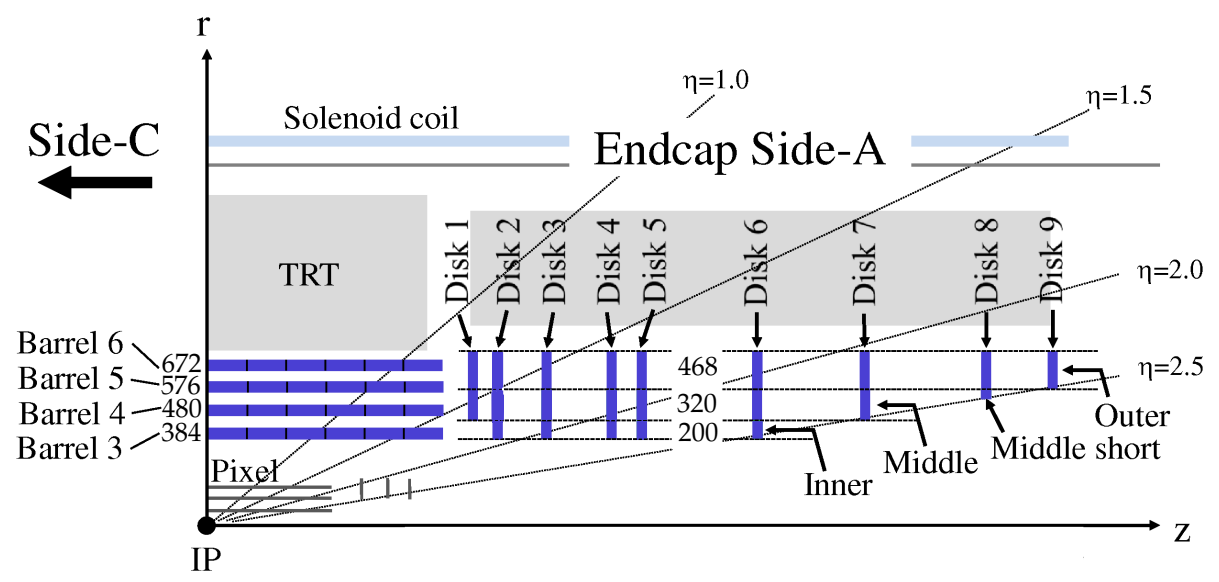

Figure 1: Cross section of one quadrant of the ATLAS Inner detector, showing the number of SCT modules in the four barrel layers and three rings of the End-caps.

The expected fluences for an integrated luminosity of $1 \mathrm{fb}^{-1}$ for the four barrel layers and three regions of the End-cap (as defined in Table 2) are given in Table 3. These values refer to silicon and the NIEL [5] hypothesis has been used to scale the results to an equivalent fluence of $1 \mathrm{MeV}$ neutrons.

Table 3: FLUKA predictions for $1 \mathrm{MeV}$ neutron equivalent fluences in the SCT using the latest geometry description of the ATLAS detector in 2017. The numbers in brackets give the range of fluences within the given regions. Statistical uncertainties are less than $1 \%$. The systematic uncertainty is estimated to be $30 \%$ [17]. The systematic uncertainties are very strongly correlated region to region. The minimum-bias $p p$ events are simulated with ATLAS tuned Pythia8 at $13 \mathrm{TeV}$ centre of mass energy and a predicted inelastic cross section of $78.4 \mathrm{mb}$. Particle tracking and interactions with material are simulated with the FLUKA 2011 code using the latest geometry description of the Run 2 ATLAS detector. The radiation backgrounds originating from protonproton collisions are symmetric in $\pm z$ in the inner detector.

\begin{tabular}{|l|l|}
\hline Sub-detector & fluence $\left(10^{11} \times 1 \mathrm{MeVn}_{\mathrm{eq}} / \mathrm{cm}^{2}\right) / \mathrm{fb}^{-1}$ \\
\hline Barrel layer 3 & $2.52(2.39-2.70)$ \\
Barrel layer 4 & $2.00(1.90-2.16)$ \\
Barrel layer 5 & $1.67(1.59-1.80)$ \\
Barrel layer 6 & $1.44(1.37-1.55)$ \\
End-cap region 1 & $1.69(1.52-1.89)$ \\
End-cap region 2 & $2.11(1.89-2.44)$ \\
End-cap region 3 & $2.68(2.43-2.86)$ \\
\hline
\end{tabular}




\section{In situ measurements at the LHC}

The in situ measurements of the radiation damage for the VCSEL ( $p-i-n$ diodes) will be described in section 4.1 (4.2). The results for the VCSELs and $p-i-n$ diodes will be given separately for the 4 barrel layers of the SCT and the three regions of the End-cap SCT (see Table 2). The results will be shown separately for the two SCT End-caps as an additional consistency check as the expected fluences are identical for the two End-caps. The results will be compared to those obtained in radiation damage studies prior to LHC operation in section 4.3.

\subsection{VCSELS}

The optical links sending data from the SCT to the counting room use a Non Return to Zero (NRZ) coding. When a link is sending a ' 0 ' the drive current is $\sim 1 \mathrm{~mA}$ and it is increased to $\sim 10 \mathrm{~mA}$ when sending a ' 1 '. Therefore over the running period, the on-detector VCSELs should experience long annealing periods with a drive current of $10 \mathrm{~mA}$. If necessary to increase the annealing rate, the drive current could be increased to $20 \mathrm{~mA}$. The light outputs of the on-detector VCSELs have been monitored by measuring the photo-currents in the off-detector opto-electronics [18]. This has been performed periodically in special scans. In one of these scans, a fixed pattern of data (consisting of a $20 \mathrm{MHz}$ clock signal) is sent via the on-detector links to the counting room. The light is received in 12 way $p-i-n$ arrays in the Back of Crate (BOC) cards [18] and the total photo-current is measured. In these scans one channel at a time out of the 12 sends this data and the other channels send no data. The measured photo-currents corresponding to each on-detector VCSEL were measured periodically as described in section 4 . The total number of VCSEL channels used in this study is given in Table 4.

Table 4: Number of VCSEL channels used for the measurements. The number of channels used in each measurement varied slightly and the numbers give the range.

\begin{tabular}{|r|c|c|}
\hline \multicolumn{1}{|c|}{ Object } & Active VCSELs & Total VCSELs \\
\hline Barrel 3 & $731-732$ & 768 \\
4 & $933-936$ & 960 \\
5 & $1130-1134$ & 1152 \\
6 & $1299-1300$ & 1344 \\
A side region 1 & $556-556$ & 704 \\
region 2 & $450-450$ & 608 \\
region 3 & $424-424$ & 664 \\
C side region 1 & $644-644$ & 704 \\
region 2 & $444-444$ & 608 \\
region 3 & $370-370$ & 664 \\
\hline
\end{tabular}

The measured photo-currents for each VCSEL number $i$, in scan $j, I(i, j)$ was normalised to the value measured before the start of the run, $I(i, 0)$ to give a normalised value, $R(i, j)=I(i, j) / I(i, 0)$. The values of $R(i, j)$ for a given scan $j$ were averaged over regions of the detector which are expected 
to receive similar fluences. The RMS deviations about the mean values of the samples contributing to the given mean were also computed. Then using the central limit theorem, for $N$ channels in the sample, the uncertainty on the mean is given by RMS $/ \sqrt{N}$. There were a few outliers caused by a problem with the procedure to measure the photo-current. To eliminate these outliers, values of $R(i, j)$ which were more than $4 \sigma$ from the mean for the scan $j$ were excluded from the fits ${ }^{5}$. The normalised photo-currents corresponding to the four layers of the barrel section of the SCT are shown as a function of integrated luminosity in fig. 2 and equivalent plots are shown for the three rings of the SCT End-cap A and C regions in fig. 3 and fig. 4. Linear fits were made to these plots and the resulting slope values are given in Table 5.

Table 5: Results of fits for radiation damage for the on-detector VCSELs for the four layers of the barrel and three regions of the End-cap disks. Linear fits were made for the normalised photocurrent $R$ versus luminosity. The gradients were also normalised to fluences using the measured luminosity and the expected fluences.

\begin{tabular}{|l|l|l|}
\hline Sub-detector & gradient $\left(10^{-2} \% / 100 \mathrm{fb}^{-1}\right)$ & gradient $\left(10^{-3} \% /\left(10^{11} \times\left(1 \mathrm{MeVn}_{\mathrm{eq}} / \mathrm{cm}^{2}\right)\right)\right.$ \\
\hline Barrel 3 & $-3.03 \pm 0.14$ & $-12.0 \pm 0.6$ \\
Barrel 4 & $-1.76 \pm 0.12$ & $-8.8 \pm 0.6$ \\
Barrel 5 & $-1.60 \pm 0.10$ & $-9.6 \pm 0.6$ \\
Barrel 6 & $-1.07 \pm 0.10$ & $-7.4 \pm 0.7$ \\
\hline End-cap A & & \\
A side region 1 & $-2.09 \pm 0.16$ & $-12.4 \pm 0.9$ \\
A side region 2 & $-1.81 \pm 0.16$ & $-8.5 \pm 0.8$ \\
A side region 3 & $-2.12 \pm 0.15$ & $-7.9 \pm 0.5$ \\
\hline End-cap C & & \\
C side region 1 & $-2.00 \pm 0.16$ & $-11.8 \pm 0.9$ \\
C side region 2 & $-1.58 \pm 0.17$ & $-7.5 \pm 0.8$ \\
C side region 3 & $-2.16 \pm 0.19$ & $-8.1 \pm 0.7$ \\
\hline
\end{tabular}

The results of these measurements are summarised in a plot of the change in VCSEL output versus the fluence (see fig. 5). A linear fit was made, but the compatibility of the data with the fit is very poor as can be assessed by the value of $\chi^{2} / \mathrm{DOF}=65.9 / 8$. The probability of randomly obtaining a larger value of $\chi^{2}$ for the given number of DOF is $3 \times 10^{-11}$. It is interesting to note that the results for End-cap A and End-cap C are consistent within each region, suggesting that the discrepancy is not a statistical effect. The largest outlier from the fit is the point corresponding to barrel 3. If this data point is removed, the quality of the fit is improved but still very poor, with a value of $\chi^{2} / \mathrm{DOF}=31.6 / 7$. The probability of randomly obtaining a larger value of $\chi^{2}$ for the given number of DOF is $3 \times 10^{-5}$.

These measurements can be compared to the radiation damage that would be expected using the studies with $30 \mathrm{MeV}$ protons (see section 2). Assuming that the threshold current and slope efficiency change linearly with fluence and ignoring annealing, the expected gradient would be approximately $-17 \times 10^{-3} \% /\left(10^{11}\left(1 \mathrm{MeVn}_{\mathrm{eq}}\right) / \mathrm{cm}^{2}\right)$. Comparing to the results after one week annealing at a

${ }^{5}$ This procedure was iterated twice to be ensure that all outliers were eliminated. 

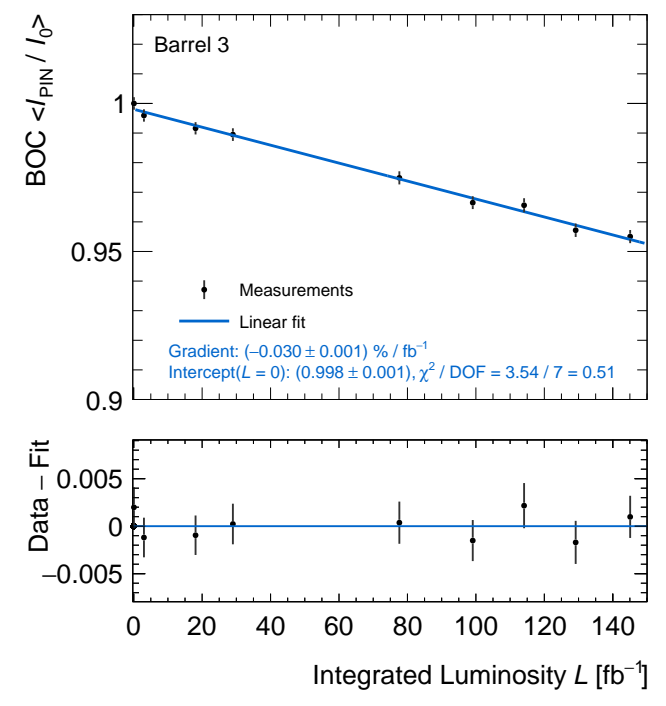

(a) Barrel 3.
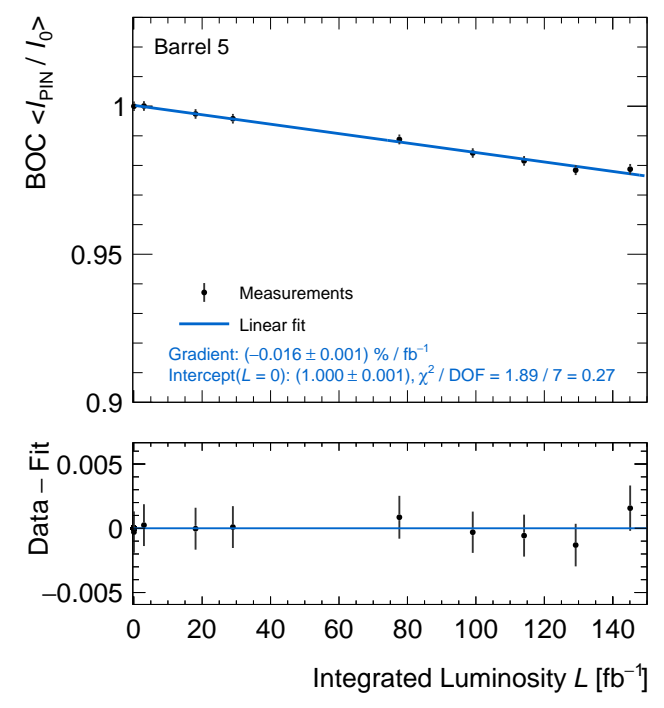

(c) Barrel 5 .
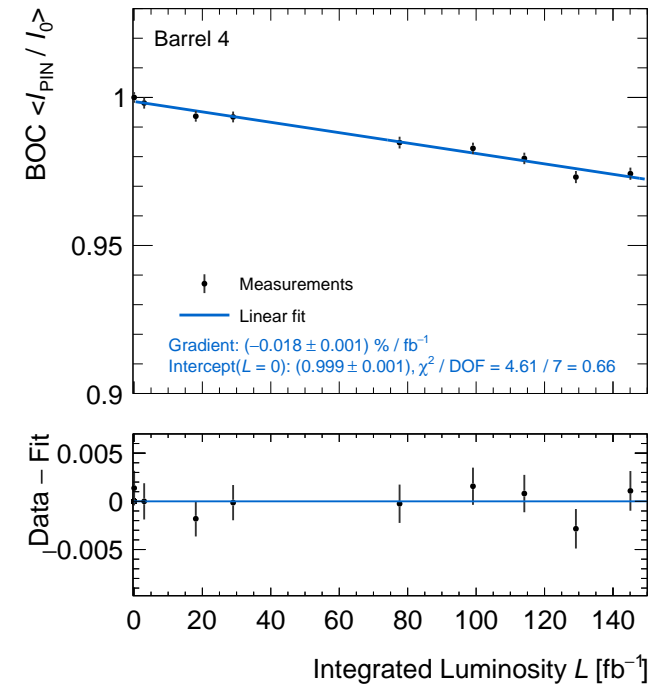

(b) Barrel 4.
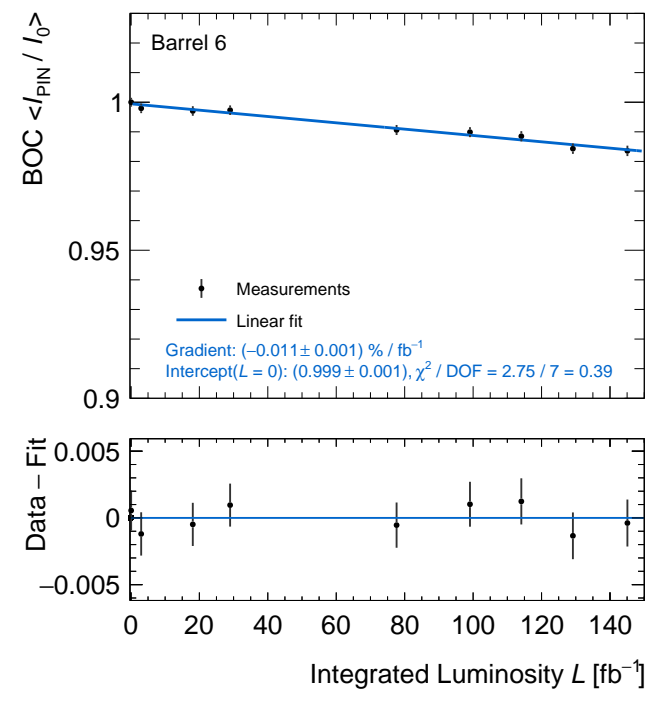

(d) Barrel 6.

Figure 2: On-detector VCSELs: Barrels. The measured mean value of the normalised photocurrents $R$ for each scan point (see text for details) correlated against integrated luminosity (see text for details).

drive current of $10 \mathrm{~mA}$, the expected gradient would be $-6 \times 10^{-3} \% /\left(10^{11}(1 \mathrm{MeVn}\right.$ eq $\left.) / \mathrm{cm}^{2}\right)$. The SCT on-detector data links use Non-Return to Zero (NRZ) coding. The VCSEL drive current when sending a ' 1 ' was $10 \mathrm{~mA}$ and approximately $1 \mathrm{~mA}$ when sending a ' 0 '. Therefore the annealing is dominated by the total time for which the detectors were sending a ' 1 '. In SCT operation during 2016, the total time for which the on-detector VCSELs were transmitting a ' 1 ' was approximately 6 weeks, hence we would expect more annealing than the one week used in the $30 \mathrm{MeV}$ proton studies. The data would therefore be expected to lie above the line corresponding to an annealing time of one week, whereas all the data points lie below this line. The extrapolation from the test beam 


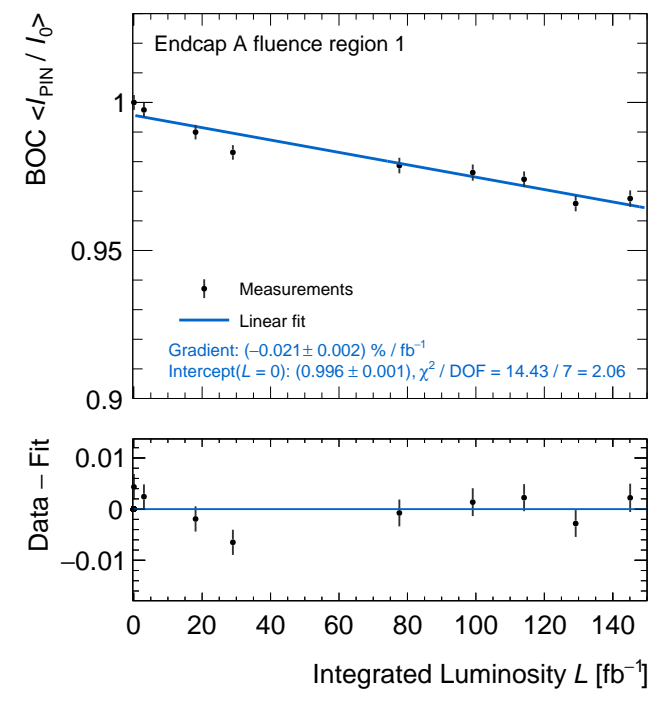

(a) End-cap region 1.
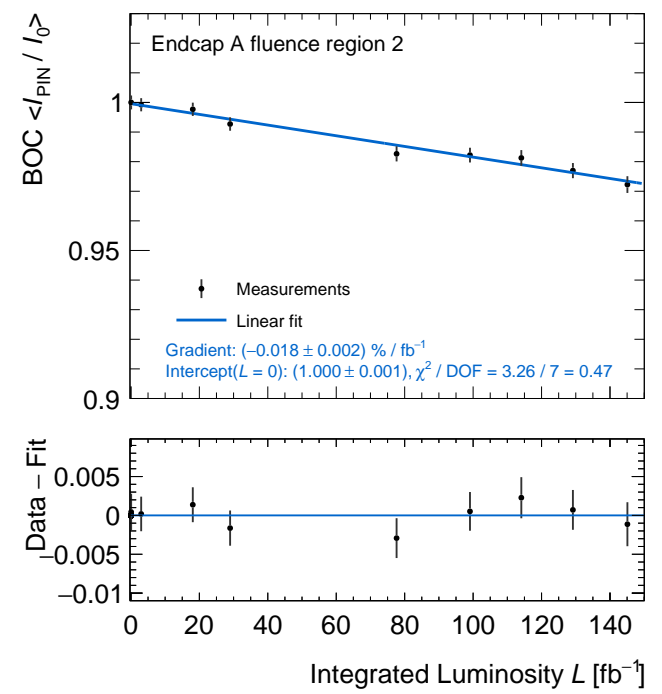

(b) End-cap A region 2.

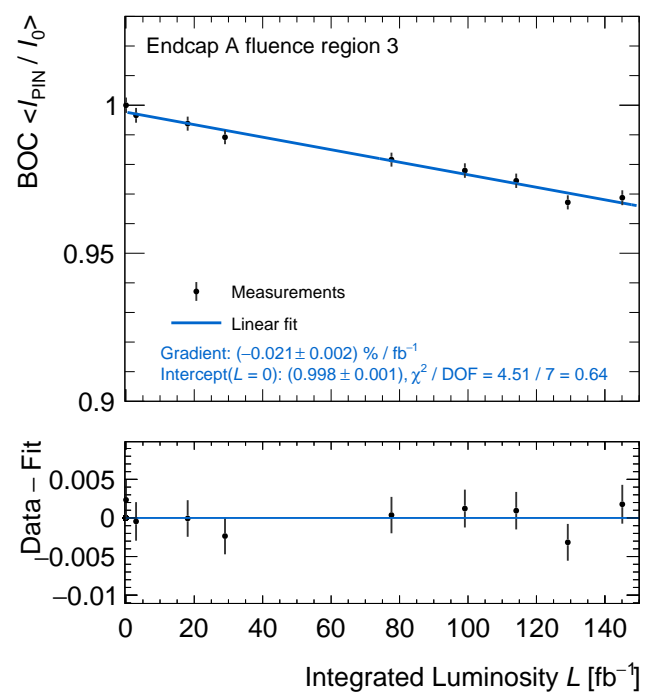

(c) End-cap A region 3.

Figure 3: On-detector VCSELs: End-cap A. The measured mean value of the normalised photocurrents $R$ for each scan point (see text for details) correlated against integrated luminosity (see text for details).

results to the in situ analysis has several sources of systematic uncertainties. The most significant uncertainties arise from the fluence calculations and the NIEL scaling hypothesis for GaAs. The measured in situ damage is therefore larger than that expected from the previous test beam studies. Extrapolating the results to the end of life for the worst region of the SCT, the decrease in optical power would be $(12 \pm 1) \%$, which can be accommodated in the links power budget [2]. If necessary the VCSEL on-current could be increased to $20 \mathrm{~mA}$ to accelerate the annealing. 

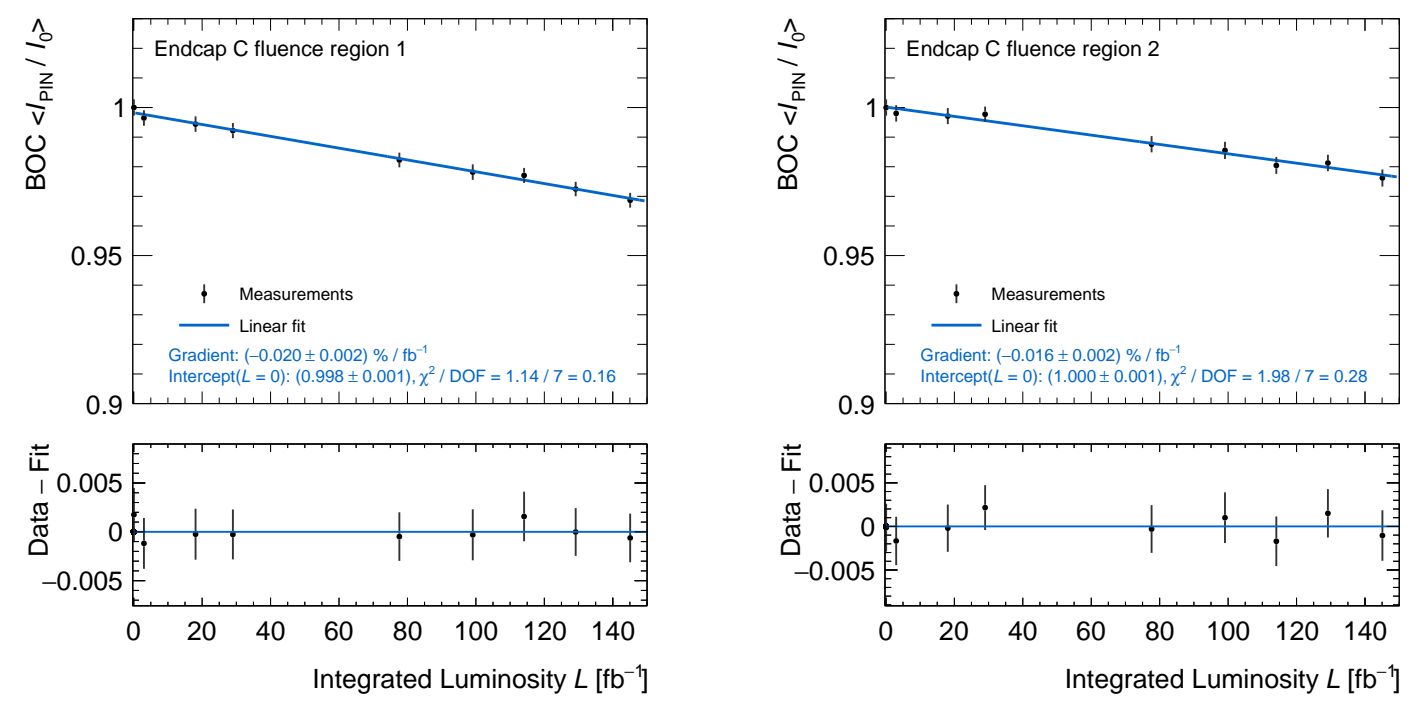

(a) End-cap C region 1.

(b) End-cap C region 2.
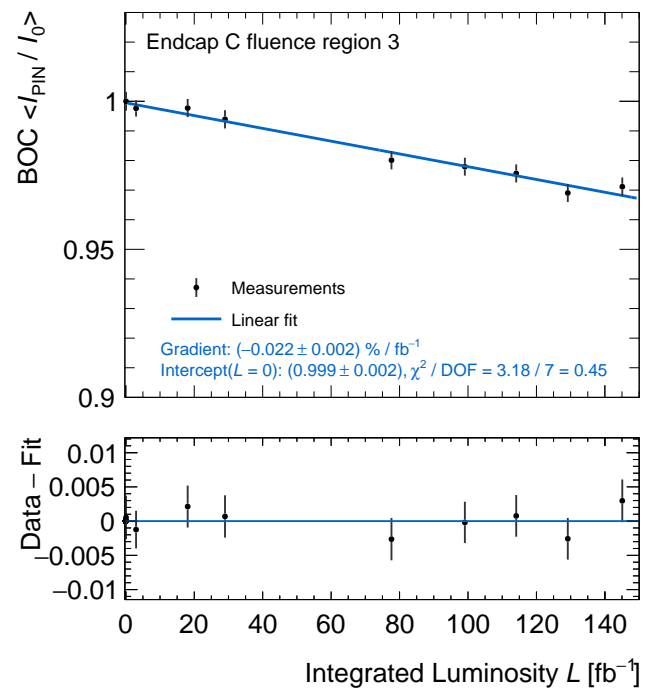

(c) End-cap C region 3.

Figure 4: On-detector VCSELs: End-cap C. The measured mean value of the normalised photocurrents $R$ for each scan point (see text for details) correlated against integrated luminosity (see text for details). 


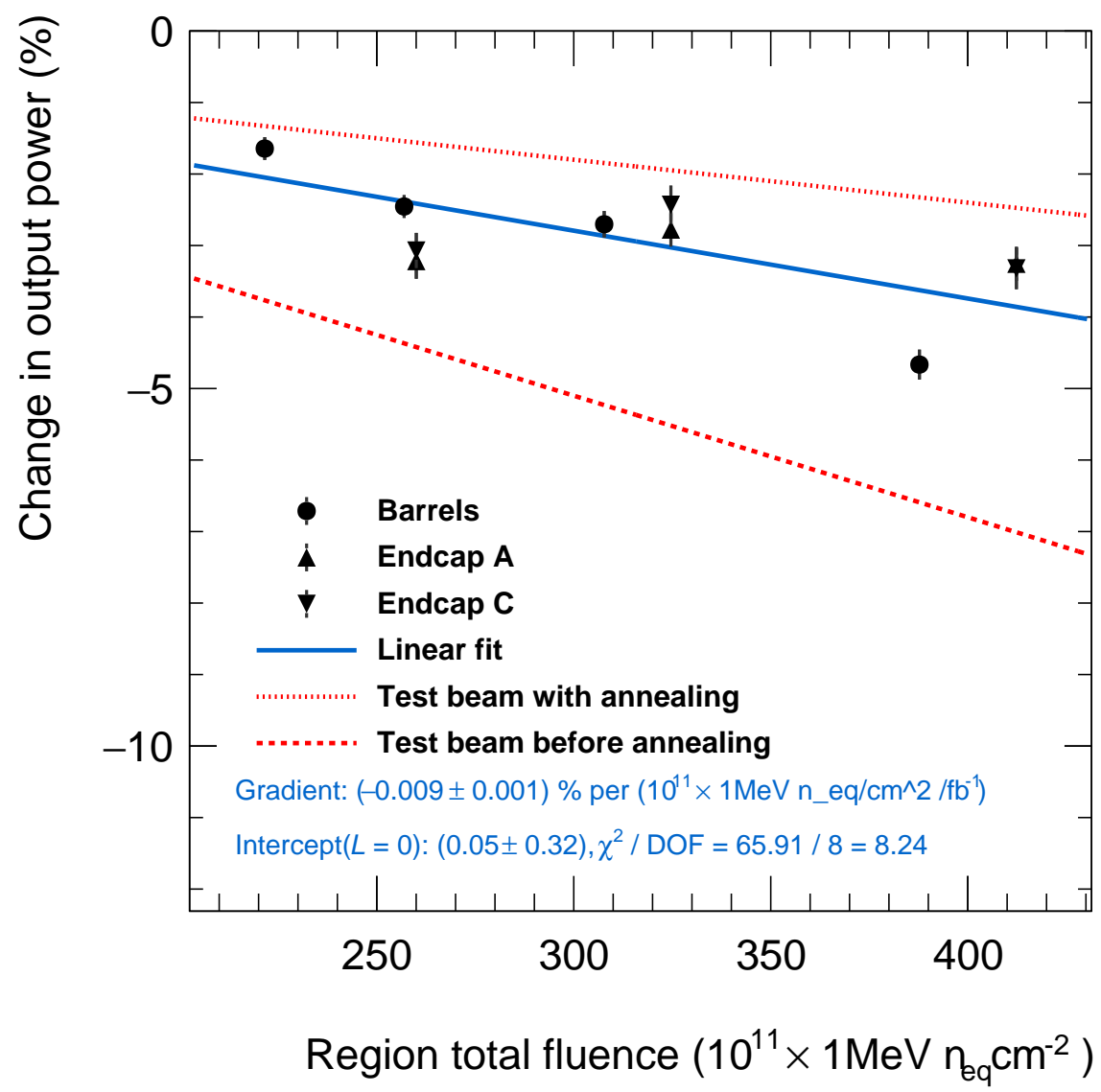

Figure 5: Plot of the change in VCSEL output versus fluence for the different regions of the SCT. 


\section{$4.2 p-i-n$ Diodes}

The responsivity of the on-detector $p-i-n$ diodes has been monitored while the detector is in operation by measuring the photo-currents. These currents are measured using the power supply in the counting room which provides the bias voltage to these $p-i-n$ diodes. The 'pedestal' values are measured during special runs with no light being sent from the off-detector VCSELs and these pedestal values are then subtracted from the measured currents while light is being sent. The currents are measured every minute during the periods in which the detector was operational. Measurements of the photo-currents are given in section 4.2.1 and measurements of the depletion voltage in section 4.2.2.

\subsubsection{Photo-currents}

Although the photo-currents in the on-detector $p-i-n$ diodes were measured from the start of SCT operation, the lack of stability of the off-detector VCSELs [19] prevented any meaningful measurements for the $p-i-n$ diodes to be made. The original type of VCSELs used in the offdetector electronics were replaced for LHC run 2.6 The stability of the new VCSELs was found to be excellent and therefore accurate measurements of the on-detector $p-i-n$ diodes could be performed from 2015 onwards. However the luminosity in the 2015 period was too low to make a reliable measurement of the radiation damage, therefore the results in this paper come from the 2016, 2017 and 2018 data.

The mean value of the $p-i-n$ diode photo-current for each channel after pedestal subtraction (see section 4) in each 24 hour period was used for the analysis ${ }^{7}$. The total number of $p-i-n$ diodes used in this study is given in Table $6 .^{8}$

Table 6: Number of modules for the $p-i-n$ diode photo-current measurements

\begin{tabular}{|r|c|c|c|}
\hline \multicolumn{1}{|c|}{ Object } & Active modules (swaps removed) & Active modules & Total modules \\
\hline Barrel 3 & $371-373$ & $371-373$ & 384 \\
4 & $470-472$ & $470-472$ & 480 \\
5 & $552-554$ & $552-554$ & 576 \\
6 & $660-664$ & $660-664$ & 672 \\
A-side fluence region 1 & $293-294$ & $344-346$ & 352 \\
2 & $197-198$ & $247-248$ & 304 \\
C-side fluence region 1 & $189-189$ & $285-287$ & 344 \\
2 & $330-333$ & $346-350$ & 352 \\
3 & $181-185$ & $245-249$ & 304 \\
\hline
\end{tabular}

${ }^{6}$ Using VCSELs from AOC and the Reflex Photonics LightABLE engine.

${ }^{7}$ The averaging procedure ignores pedestal readings to avoid being biased by periods in which the off-detector electronics was not operational.

${ }^{8}$ Some fibres were swapped in the off-detector BOC crates between the 2016 and 2017 runs in order to optimise the bandwidth. Therefore the corresponding channels are excluded from this analysis. 
Table 7: Results of fits for the normalised $p-i-n$ diode currents as a function of time during no-beam operation. The gradients in 2016, 2017 and 2018 were measured over 52, 19 and 28 days before data taking, respectively.

\begin{tabular}{|r|r|l|}
\hline Crate & Fit gradient $\left(10^{-4}\right.$ days $\left.^{-1}\right)$ & $\chi^{2} /$ DOF \\
\hline & $\mathbf{2 0 1 6}$ & \\
\hline 0 & $-3.2 \pm 0.4$ & 15.5 \\
1 & $-2.2 \pm 0.3$ & 6.5 \\
2 & $-1.5 \pm 0.2$ & 3.9 \\
3 & $-1.6 \pm 0.2$ & 5.6 \\
4 & $1.9 \pm 0.3$ & 4.9 \\
5 & $0.6 \pm 0.2$ & 8.2 \\
6 & $-2.7 \pm 0.3$ & 8.3 \\
7 & $-2.0 \pm 0.3$ & 10.9 \\
\hline & $\mathbf{2 0 1 7}$ & \\
\hline 0 & $1.0 \pm 0.7$ & 4.6 \\
1 & $2.6 \pm 1.0$ & 5.5 \\
2 & $3.0 \pm 0.7$ & 4.3 \\
3 & $1.2 \pm 0.4$ & 4.7 \\
4 & $2.1 \pm 0.5$ & 5.9 \\
5 & $2.2 \pm 0.5$ & 3.2 \\
6 & $2.6 \pm 0.4$ & 0.8 \\
7 & $2.4 \pm 0.6$ & 2.5 \\
\hline & $\mathbf{2 0 1 8}$ & \\
\hline 0 & $2.0 \pm 0.5$ & 0.5 \\
1 & $0.9 \pm 0.4$ & 0.2 \\
2 & $2.1 \pm 0.5$ & 0.3 \\
3 & $0.8 \pm 0.3$ & 0.04 \\
4 & $1.4 \pm 0.2$ & 0.02 \\
5 & $1.6 \pm 0.3$ & 0.01 \\
6 & $2.5 \pm 0.5$ & 0.2 \\
7 & $3.3 \pm 0.5$ & 0.1 \\
\hline & & \\
& & \\
& & \\
& &
\end{tabular}

The $p-i-n$ diode photo-currents were measured before the start of the runs in order to determine the stability of the system. Linear fits were performed to the normalised $p-i-n$ diode photo-current as a function of time (see Appendix A for all the plots). The normalisation was performed using the value of the $p-i-n$ diode current at the start of data taking for that year. The results of the fits are summarised in table 7. The small values of the slopes are a measure of the stability of the system. No significant trend can be identified but the RMS spread of the slopes will be used as one source of systematic uncertainty in the final results.

The measured $p-i-n$ diode photo-currents as a function of luminosity $(L)$ for each channel $(i)$ 
$I_{\mathrm{PIN}}(L, i)$ were normalised by the value at the start of 2016 data taking $I_{\mathrm{PIN}}(0, i)$. The values of $I_{\mathrm{PIN}}(L, i) / I_{\mathrm{PIN}}(0, i)$ were averaged over the channels $i$ over the same regions of the detector as used for the analysis of the on-detector VCSELs (see section 4.1). The central limit theorem was used to determine the uncertainty on the mean values in an equivalent way to that used in section 4.1. The measured mean normalised $p-i-n$ diode photo-currents data versus luminosity delivered by the LHC from 3 May 2016 to 30 October 2016, 5 May 2017 to 17 November 2017 and 17 April 2018 to 25 October 2018 are shown for the barrels in fig. 6 and for the End-caps in fig. 7 and fig. 8. In most cases the continuity between the 2016 and 2017 periods and 2017 and 2018 periods is very good despite the detector having been switched off during the Year End Technical Stops. However there are some cases (e.g. barrel 6) for which a clear discontinuity was observed and in these cases the 2017 (2018) data are scaled to remove this discontinuity. The data show a clear decrease with luminosity. The data are clearly inconsistent with a linear decrease but can be fitted by functions of the form

$$
\left\langle I_{\mathrm{PIN}}(L) / I_{\mathrm{PIN}}(0)\right\rangle=R_{0} \exp [f(0)-f(L)]+R_{\infty},
$$

where the constraint $R_{0}+R_{\infty}=1$ is used and the function should decrease monotonically to $R_{\infty}$ as $L \rightarrow \infty$. A simple empirical function was used

$$
f(L)=\left(-A /\left(B+L^{2}\right)+L / L_{0}\right),
$$

and $A, B, L_{0}$ are free parameters in the fit. The parameter $R_{\infty}$ gives the asymptotic value for the fractional asymptotic decrease in the $p-i-n$ diode current. Two alternative simple empirical fit functions which also have asymptotic values were used

$$
f(L)=L / L_{0}+\left(L / L_{1}\right)^{0.75}+\left(L / L_{2}\right)^{0.5},
$$

and

$$
f(L)=\left(L / L_{0}\right)^{m} .
$$

These alternative functional forms gave worse quality fits and were only used to estimate an additional systematic uncertainty in the final result. The fitted values are given in Table 8 . The values of $\chi^{2} / \mathrm{DOF}$ for all these fits are very low, which suggests that there are strong correlations in the data points. This can arise as an artefact of the normalisation procedure as for each channel every data point is normalised to the same initial value. This will tend to over-estimate the statistical errors but as the final results are dominated by systematic errors, the importance of this effect is greatly reduced. An alternative procedure was tried in which the initial value for the $p-i-n$ diode current for each channel was averaged over 10 days, rather than one day but this did not significantly impact the $\chi^{2} / \mathrm{DOF}$ values.

The values for $R_{\infty}$ given in Table 8 can be used to determine a weighted mean estimate for this parameter. Each region has a statistical uncertainty from the fit, a stability uncertainty, and a parametrisation uncertainty taken as the largest of the differences between the $R_{\infty}$ values of the alternative parametrisations and the central one. The stability uncertainty is uncorrelated between regions whereas the parametrisation uncertainty is fully correlated. The stability uncertainty is calculated as the product of the RMS stability error and the number of days of beam operation. The overall result is calculated as the weighted mean over the regions. There are two contributions 
Table 8: Tabulated values for the fitted values of $R_{\infty}$ (see text for details) for the different regions of the SCT.

\begin{tabular}{|r|c|c|c|c|c|}
\hline Object & $R_{\infty}$ & $\begin{array}{l}\text { Statistical } \\
\text { uncertainty }\end{array}$ & $\begin{array}{l}\text { Stability } \\
\text { uncertainty }\end{array}$ & $\begin{array}{c}\text { Parametrisation } \\
\text { uncertainty }\end{array}$ & $\begin{array}{l}\text { Total } \\
\text { uncertainty }\end{array}$ \\
\hline Barrel 3 & 0.880 & 0.001 & 0.083 & 0.003 & 0.083 \\
Barrel 4 & 0.862 & 0.002 & 0.083 & 0.008 & 0.083 \\
Barrel 5 & 0.870 & 0.001 & 0.083 & 0.001 & 0.083 \\
Barrel 6 & 0.863 & 0.001 & 0.083 & 0.007 & 0.083 \\
A side region 1 & 0.749 & 0.004 & 0.083 & 0.033 & 0.089 \\
A side region 2 & 0.787 & 0.004 & 0.083 & 0.024 & 0.086 \\
A side region 3 & 0.759 & 0.003 & 0.083 & 0.059 & 0.102 \\
C side region 1 & 0.862 & 0.001 & 0.083 & 0.009 & 0.083 \\
C side region 2 & 0.857 & 0.003 & 0.083 & 0.016 & 0.084 \\
C side region 3 & 0.849 & 0.006 & 0.083 & 0.037 & 0.091 \\
\hline
\end{tabular}

to the uncertainty on the result; the first is calculated as a standard sum of square weights, where the weight used here excludes the parametrisation uncertainty on each region, and the second is a parametrisation uncertainty on the final result, calculated as the largest difference between two alternative parametrisations. This procedure gives a result $R_{\infty}=0.834 \pm 0.026 \pm 0.015$ where the first error is the combination of the statistical and stability uncertainties and the second is the parametrisation uncertainty. The data for the separate regions are consistent with this value, $\chi^{2} / \mathrm{DOF}=2.7 / 9$. The probability of randomly obtaining a larger value of $\chi^{2}$ for the given number of DOF is 0.976 . 


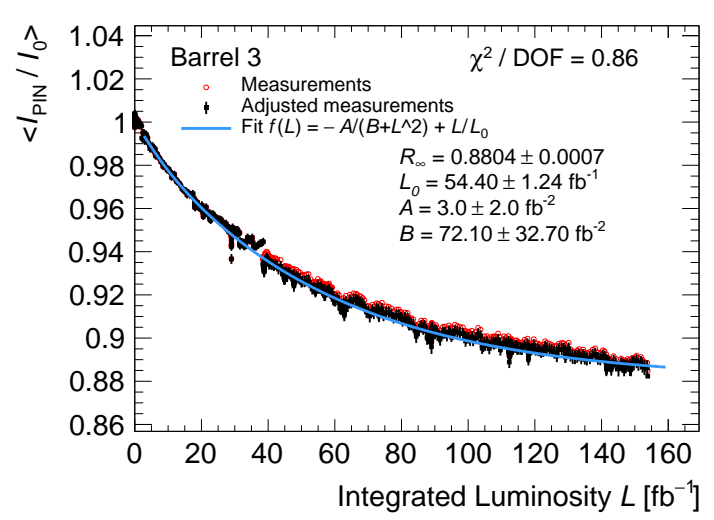

(a) Barrel 3.

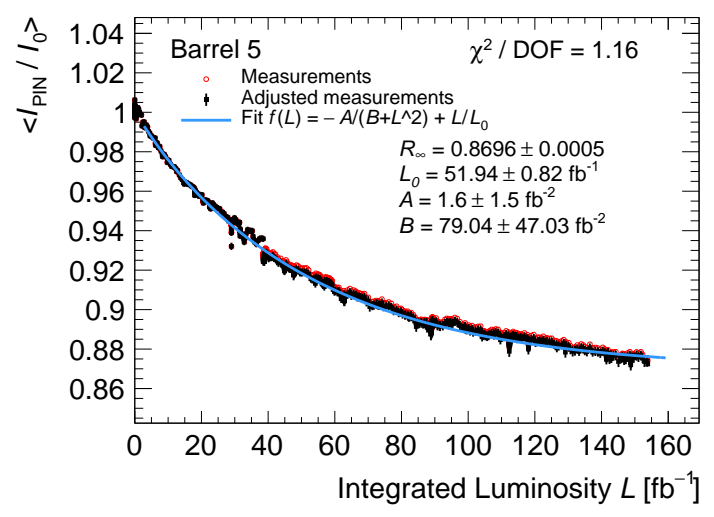

(c) Barrel 5.

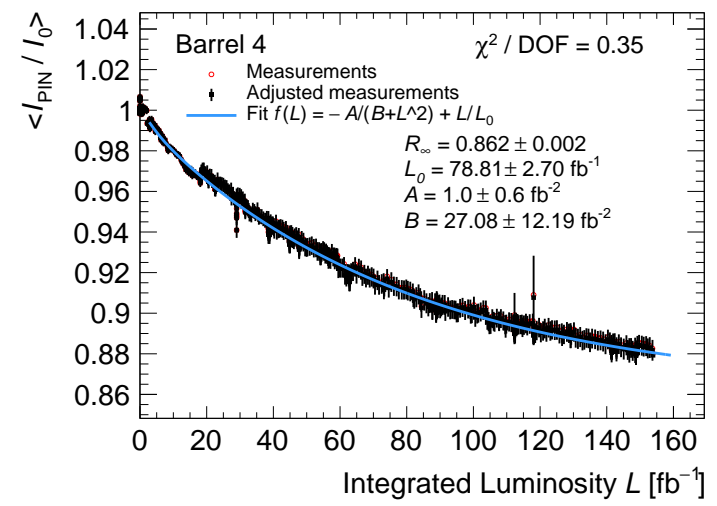

(b) Barrel 4.

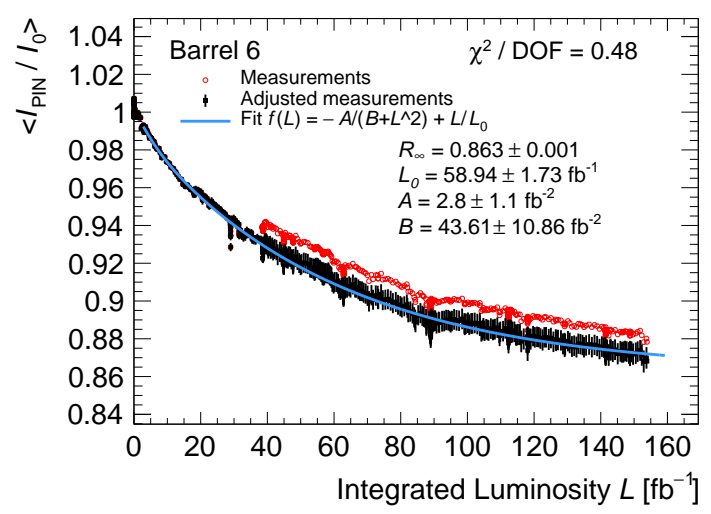

(d) Barrel 6.

Figure 6: Barrels 2016, 2017 and 2018. The $\left\langle I_{\mathrm{PIN}} / I_{0}\right\rangle$ values of each barrel layer correlated against integrated luminosity (see text for details). Each point represents the mean value for one day averaged over all modules for each layer. The value of $\left\langle I_{\mathrm{PIN}} / I_{0}\right\rangle$ is the normalised mean value of $I_{\mathrm{PIN}}$ (see text for details). The curves show results of fits to the measurements using the parametrisation labelled in each plot. 


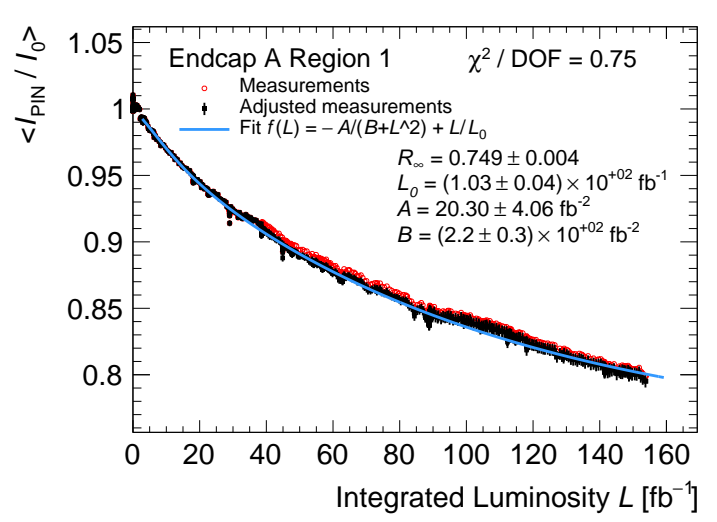

(a) End-cap A region 1.

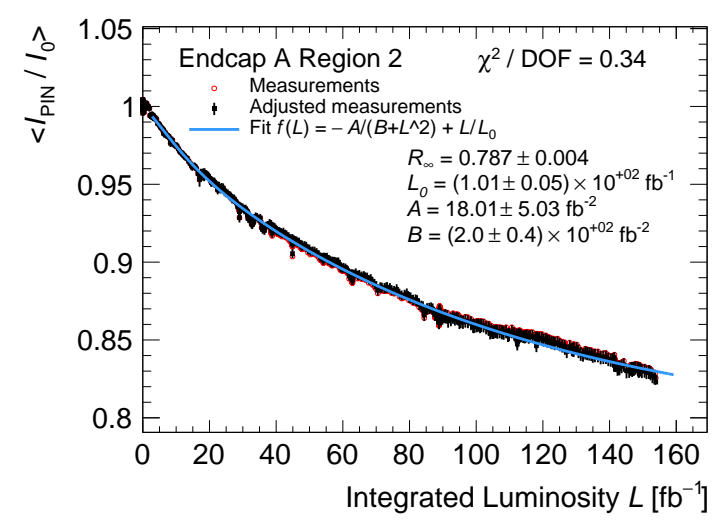

(b) End-cap A region 2.

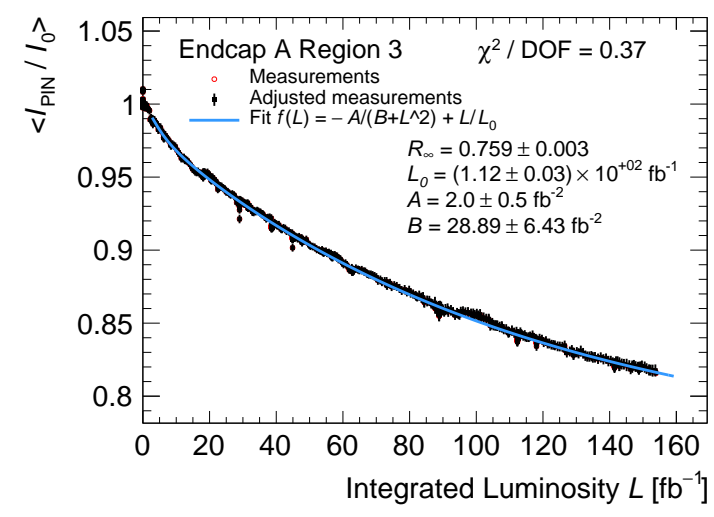

(c) End-cap A region 3.

Figure 7: End-cap A 2016, 2017 and 2018. The $\left\langle I_{\mathrm{PIN}} / I_{0}\right\rangle$ values of each disk region for End-cap A correlated against integrated luminosity (see text for details). Each point represents the mean value for one day averaged over all modules for each region. The value of $\left\langle I_{\mathrm{PIN}} / I_{0}\right\rangle$ is the normalised mean value of $I_{\text {PIN }}$ (see text for details). The curves show results of fits to the measurements using the parametrisation labelled in each plot. 


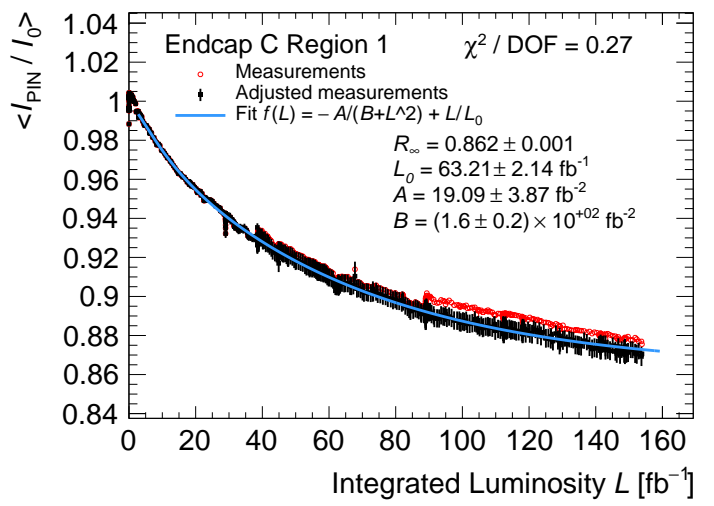

(a) End-cap C region 1.

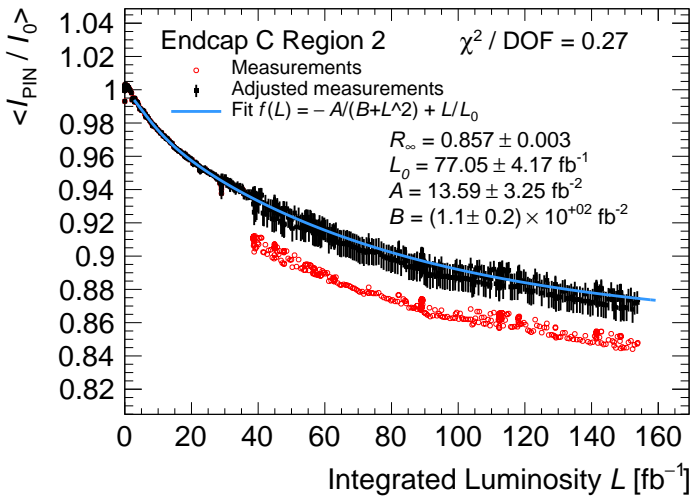

(b) End-cap C region 2.

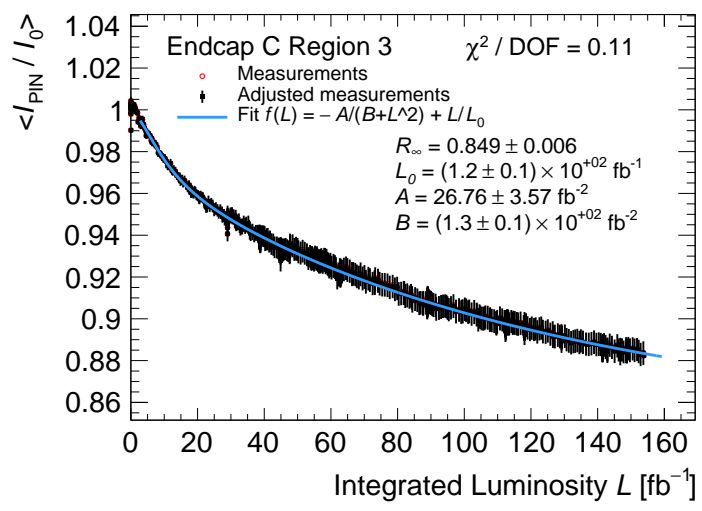

(c) End-cap C region 3.

Figure 8: End-cap C 2016, 2017 and 2018. The $\left\langle I_{\mathrm{PIN}} / I_{0}\right\rangle$ values of each disk region for End-cap C correlated against integrated luminosity (see text for details). Each point represents the mean value for one day averaged over all modules for each region. The value of $\left\langle I_{\mathrm{PIN}} / I_{0}\right\rangle$ is the normalised mean value of $I_{\text {PIN }}$ (see text for details). The curves show results of fits to the measurements using the parametrisation labelled in each plot. 


\subsubsection{Depletion Voltage}

Scans of the $p-i-n$ diode current versus bias voltage were performed to determine if there was any change in the full-depletion voltage. The scans varied the bias voltage from $1 \mathrm{~V}$ to $10 \mathrm{~V}$ in $1 \mathrm{~V}$ steps. If the $p-i-n$ diodes are fully depleted at $1 \mathrm{~V}$, then there would be no change in photo-current with bias voltage. A fit with a constant value of the photo-current was made for all channels and if these fits were acceptable (having $\chi^{2} / \mathrm{DOF}<3$ ), these channels clearly had a full-depletion voltage below $1 \mathrm{~V}$. The majority of the modules were in this category. For those cases in which there was evidence for the photo-current $\left(I_{p c}\right)$ varying with bias voltage $\left(V_{b}\right)$, an exponential fit of the form

$$
I_{p c}=I_{1}-I_{0} \cdot \exp \left(-V_{b} / V_{0}\right)
$$

was used, where $I_{0}, V_{0}$ and $I_{1}$ are free parameters to be determined by the fit. A first estimate of the full-depletion voltage $\left(V_{d e p}\right)$ was made by determining the bias voltage at which the value of $I_{p c}=0.95 I_{1}$. This raw value was then corrected to allow for the voltage drop in the chain from the power supply to the $p-i-n$ diode. The histogram of the corrected value of the depletion voltages $\left(V_{d e p}\right)$ for these channels is shown for the barrel layers, End-cap A and End-cap C in figs 9-11. The distributions are centred around a value of $0 \mathrm{~V}$, which reflects the fact that the true values of the depletion voltage are too small to be determined with this analysis. However the analysis would have detected a change in depletion voltage larger than about $1 \mathrm{~V}$.

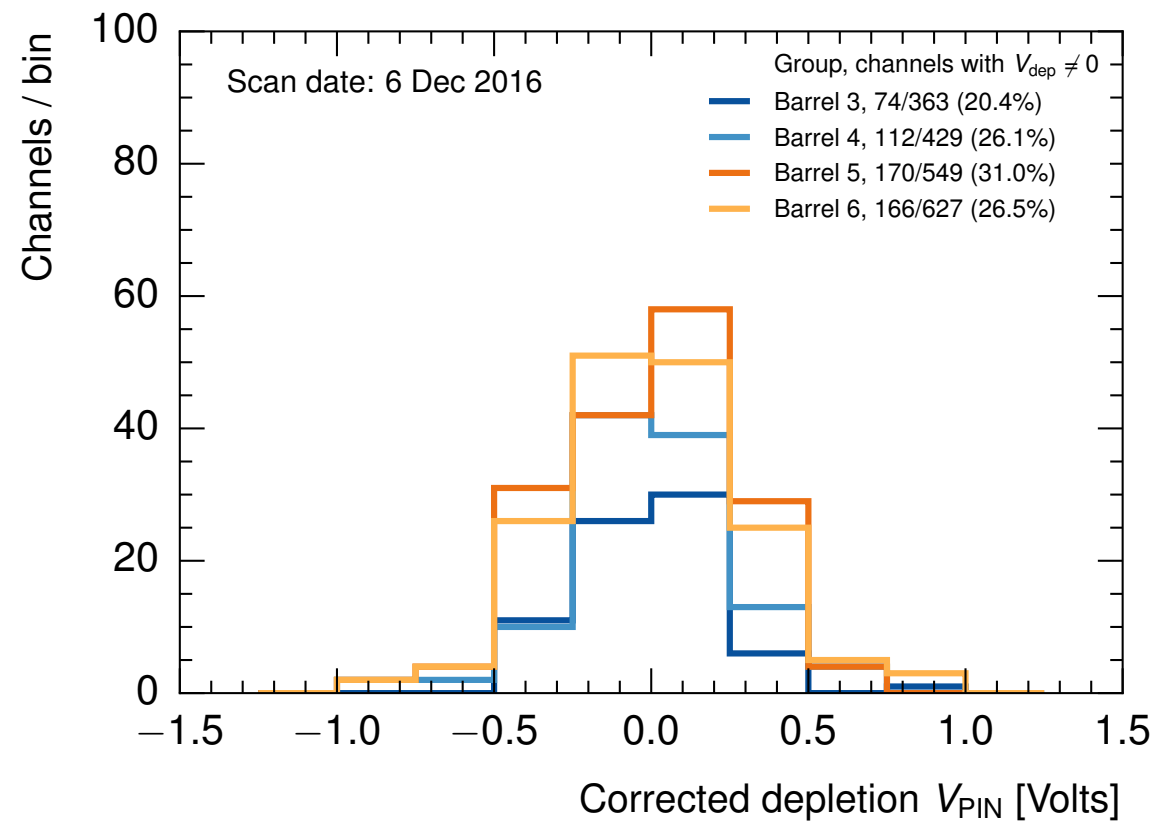

Figure 9: Histogram of the corrected $p-i-n$ diode depletion voltages from the end of data taking in 2016 for the 4 barrel layers. The sample is limited to those for which there was any evidence of a change in depletion voltage, the fraction of which is displayed in the legend. See text for definition of the corrected depletion voltage. 


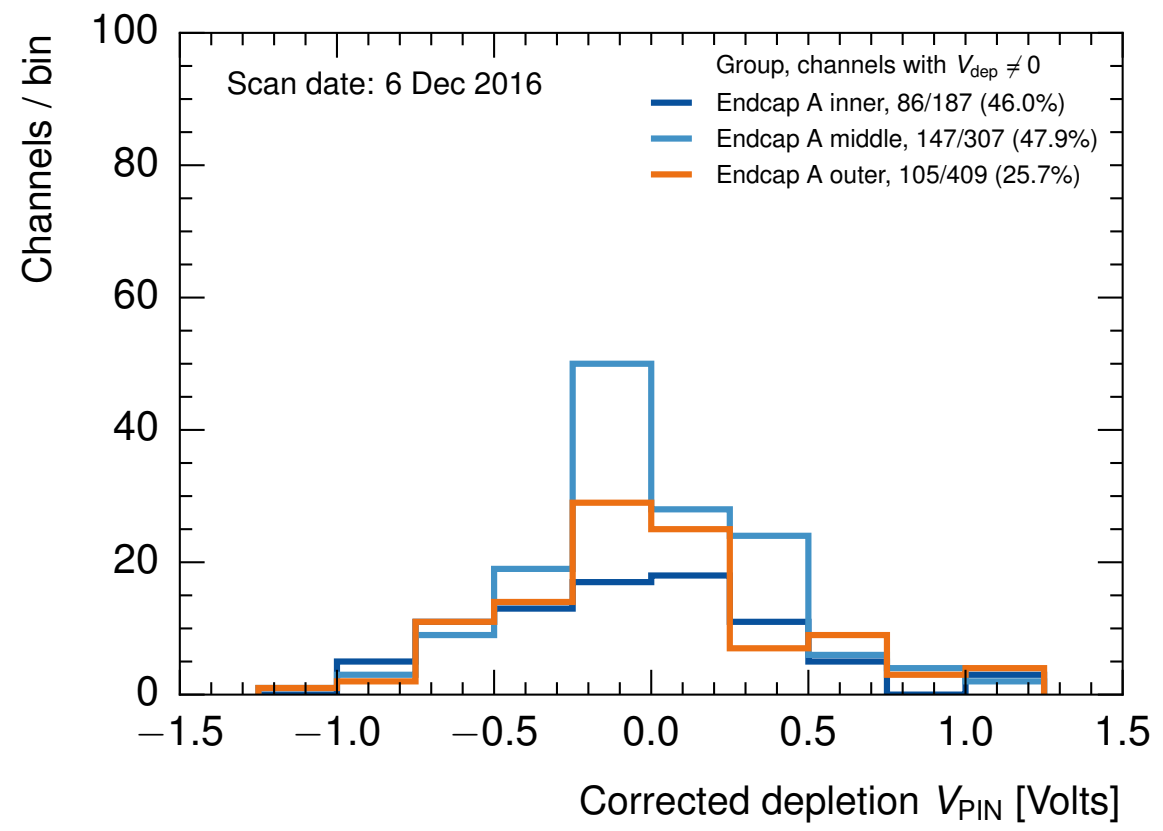

Figure 10: Histogram of $p-i-n$ diode depletion voltages from the end of data taking in 2016 for modules in End-cap A. The sample is limited to those for which there was any evidence of an increase in depletion voltage. 


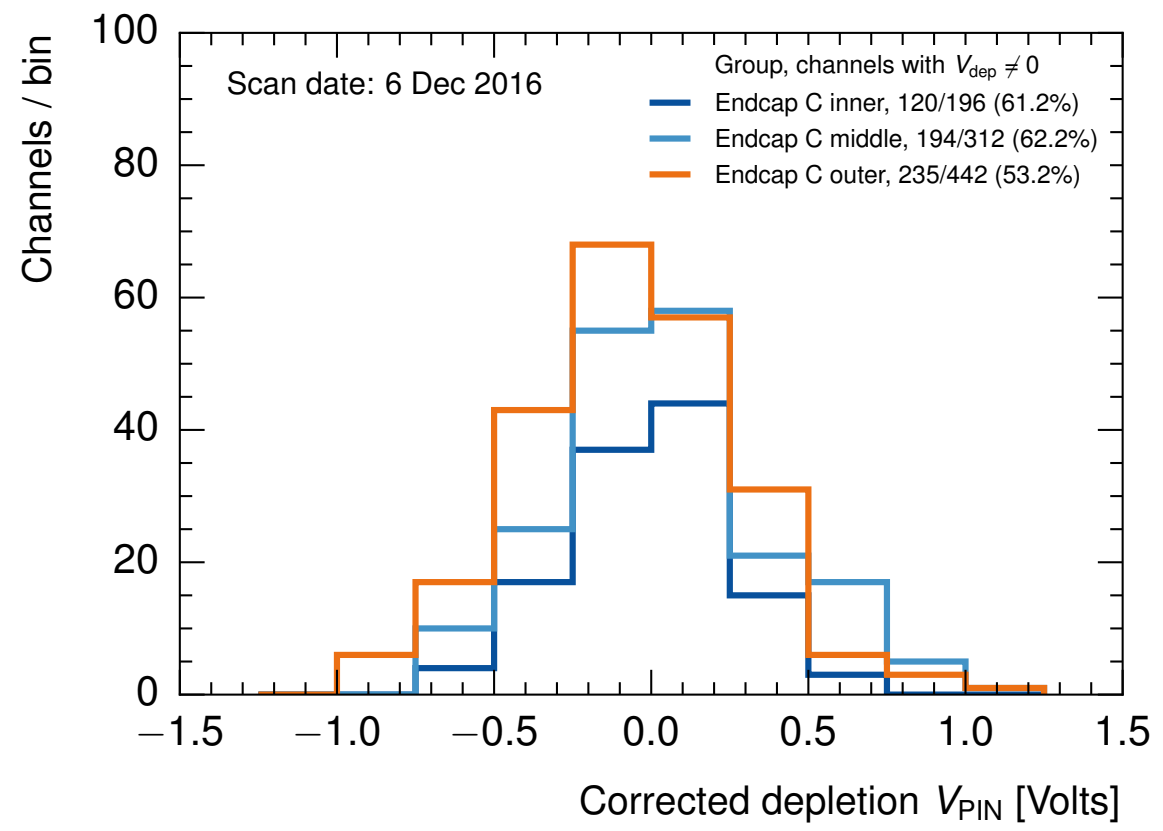

Figure 11: Histogram of $p-i-n$ diode depletion voltages from the end of data taking in 2016 for for modules in End-cap C. The sample is limited to those for which there was any evidence of an increase in depletion voltage. 


\subsection{Discussion of Results}

The on-detector VCSELs showed strong evidence for a small decrease in optical output with radiation. This observation was compared with the expectations from previous measurements with $30 \mathrm{MeV}$ proton beams assuming NIEL scaling for GaAs and found to be larger than expected. The measured decrease in optical power can be extrapolated to the full expected luminosity $\sim 350 \mathrm{fb}^{-1}$ to predict a decrease in optical power of $(12 \pm 1) \%$ for the module receiving the highest fluence. Losses of this magnitude can be accommodated within the links power budget so should not be a problem for SCT operation. If necessary, the VCSEL drive currents could be increased to $20 \mathrm{~mA}$ for a period of time to increase the beneficial annealing. However the on-detector VCSELs should continue to be monitored with increasing integrated luminosity to allow more precise comparisons with extrapolations from previous test beam studies and to try to understand the inconsistencies in the results for different regions of the detector.

The on-detector $p-i-n$ diodes showed decreases in responsivity with radiation. The mean value for the asymptotic response was determined to be $R_{\infty}==0.834 \pm 0.026 \pm 0.015$ (see sec. 4.2). In order to make a comparison with the earlier data from test beam studies it is necessary to consider the effect of the radiation damage received by the $p-i-n$ diodes during the period 2011-2015. The effective luminosity ( $\mathcal{L}_{\text {eff }}$ ) scaled to $13 \mathrm{TeV}$ operation was calculated for the 7 and $8 \mathrm{TeV}$ data sets by allowing for the relative fluences per luminosity and the results shown in Table 9.

The fit function used for the $p-i-n$ diode responsivity versus fluence (eqn 4.2) is not defined for negative fluences. Therefore the fits were performed with the luminosity offset by the total luminosity received prior to 2016 operation (see Table 9). The results of these fits are shown for the barrel in fig. 12 and for the End-caps in fig. 13 and fig. 14. These fits were then used to determine the corrected value for the asymptotic damage relative to the start of operation $\left(R_{\infty}^{\text {eff }}\right)$. The values for $R_{\infty}^{\text {eff }}$ are given in Table 10 .

Using the same procedure as in section 4.2 the weighted mean value is given by $R_{\infty}^{\text {eff }}=$ $0.731 \pm 0.027 \pm 0.046$, where the first error is the combination of the statistical and stability uncertainties (see section 4.2) and the second is the parametrisation uncertainty. The data for the different regions are consistent, $\chi^{2} / \mathrm{DOF}=8.6 / 9$. The probability of randomly obtaining a larger value of $\chi^{2}$ for the given number of DOF is 0.47 . The extrapolation procedure results in a

Table 9: Effective luminosity for LHC operation before 2016.

\begin{tabular}{|l|l|}
\hline Detector region & $\mathcal{L}_{\text {eff }}\left(\mathrm{fb}^{-1}\right)$ \\
\hline Barrel layer 3 & 23.4 \\
Barrel layer 4 & 23.1 \\
Barrel layer 5 & 22.9 \\
Barrel layer 6 & 22.7 \\
End-cap region 1 & 23.0 \\
End-cap region 2 & 22.5 \\
End-cap region 3 & 23.0 \\
\hline
\end{tabular}


Table 10: Fitted values of $R_{\infty}^{\text {eff }}$ (see text for details) for the different regions of the SCT.

\begin{tabular}{|r|c|l|l|l|l|}
\hline Object & $R_{\infty}^{\text {eff }}$ & $\begin{array}{l}\text { Statistical } \\
\text { uncertainty }\end{array}$ & $\begin{array}{l}\text { Stability } \\
\text { uncertainty }\end{array}$ & $\begin{array}{l}\text { Parametrisation } \\
\text { uncertainty }\end{array}$ & $\begin{array}{l}\text { Total } \\
\text { uncertainty }\end{array}$ \\
\hline Barrel 3 & 0.782 & 0.007 & 0.083 & 0.046 & 0.095 \\
Barrel 4 & 0.717 & 0.012 & 0.083 & 0.108 & 0.137 \\
Barrel 5 & 0.812 & 0.002 & 0.083 & 0.017 & 0.085 \\
Barrel 6 & 0.777 & 0.007 & 0.083 & 0.055 & 0.100 \\
A side region 1 & 0.636 & 0.014 & 0.083 & 0.085 & 0.120 \\
A side region 2 & 0.770 & 0.007 & 0.083 & 0.069 & 0.108 \\
A side region 3 & 0.571 & 0.009 & 0.083 & 0.303 & 0.314 \\
C side region 1 & 0.791 & 0.002 & 0.083 & 0.083 & 0.117 \\
C side region 2 & 0.791 & 0.008 & 0.083 & 0.124 & 0.149 \\
C side region 3 & 0.654 & 0.028 & 0.083 & 0.174 & 0.195 \\
\hline
\end{tabular}

relatively large uncertainty on the fit function used and therefore the error is now dominated by the systematic uncertainty associated to the choice of fit function. This result is compatible with the result measured from earlier test beam studies, of $0.71[11]^{9}$. As expected the increase in depletion voltages for the on-detector $p-i-n$ diodes was below the sensitivity for our measurement system.

The $p-i-n$ diodes were operated above full-depletion voltage therefore the decrease in responsivity can not be attributed to a decrease in the depth of the depleted region. While a fast signal will arise from drift of the free carriers created in the depleted region of the $p-i-n$ diode, there can also be a much slower signal from carrier diffusion [20] in the undepleted region (n-type silicon in the $p$-i-n structure $)^{10}$. Some of these carriers created in the $n$-type silicon will diffuse into the depleted $i$-type silicon layer, where they will then generate a signal from the resulting drift in the large electric field. The most plausible explanation for the decrease in responsivity is that the responsivity measurements are all based on DC systems and are therefore sensitive to a slow diffusion component. Any slow diffusion component will be greatly reduced by charge trapping after radiation damage and the remaining responsivity will be due to the drift signal from the carriers created in the depleted $i$-type silicon. The expected asymptotic decreases in responsivity can also be accommodated within the links power budget. However it will be important to continue to monitor the $p-i-n$ diodes to see if the radiation damage shows the expected asymptotic behaviour for all regions of the detector.

${ }^{9}$ For fluences over an order of magnitude higher than used in this study, the full depletion voltage of the $p-i-n$ diode would become larger than the $p-i-n$ diode bias voltage used. This would result in the $i$-region becoming not fully depleted and this would result in a further decrease in responsivity.

${ }^{10}$ The depth of the $i$-type silicon is $15 \mu \mathrm{m}$, which is comparable to the absorption depth in silicon for a wavelength of $850 \mathrm{~nm}$ of $10 \mu \mathrm{m}$. Therefore some of the charge carriers from the incident photon will be created in the $n$-type layer of the $p-i-n$ diode. 


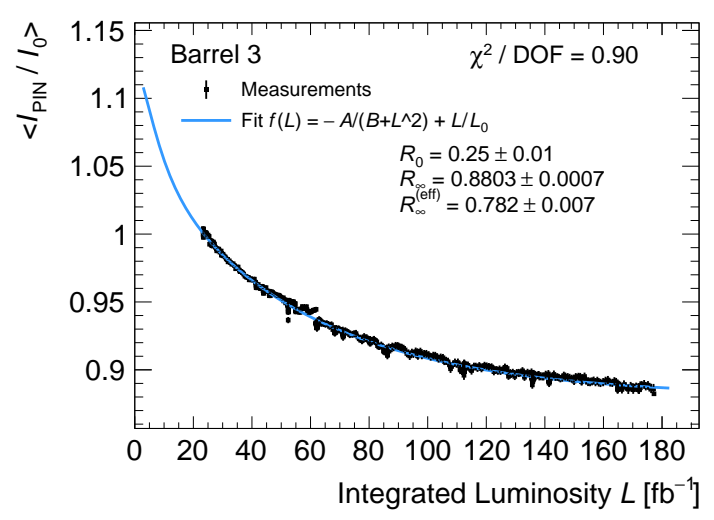

(a) Barrel 3 .

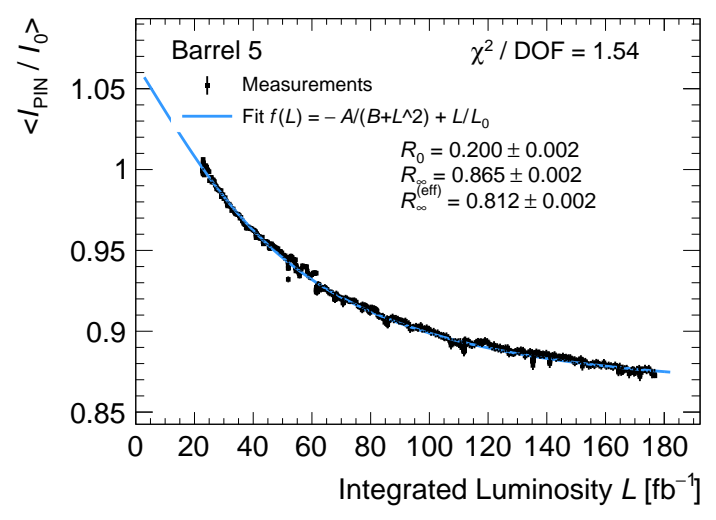

(c) Barrel 5.

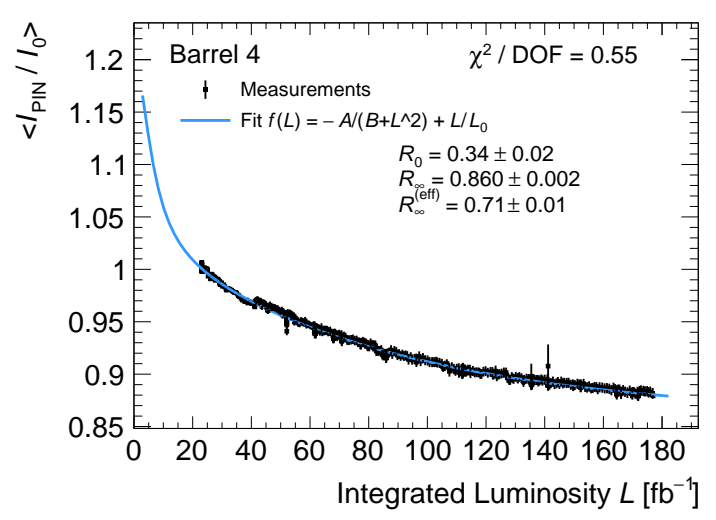

(b) Barrel 4.

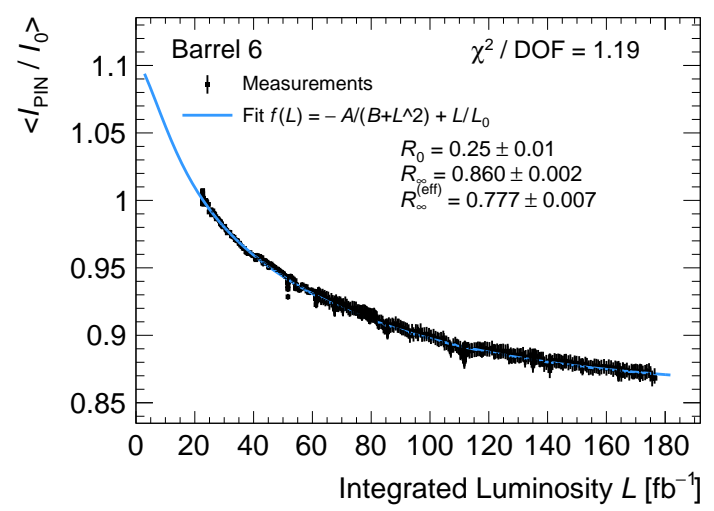

(d) Barrel 6 .

Figure 12: Barrels 2016, 2017 and 2018. The $\left\langle I_{\mathrm{PIN}} / I_{0}\right\rangle$ values of each barrel layer correlated against integrated luminosity (see text for details). The data is offset on the horizontal axis to allow for the effective luminosity delivered before 2016. Each point represents the mean value for one day averaged over all modules for each layer. The value of $\left\langle I_{\mathrm{PIN}} / I_{0}\right\rangle$ is the normalised mean value of $I_{\mathrm{PIN}}$ (see text for details). The curves show results of fits to the measurements using the parametrisation labelled in each plot. 


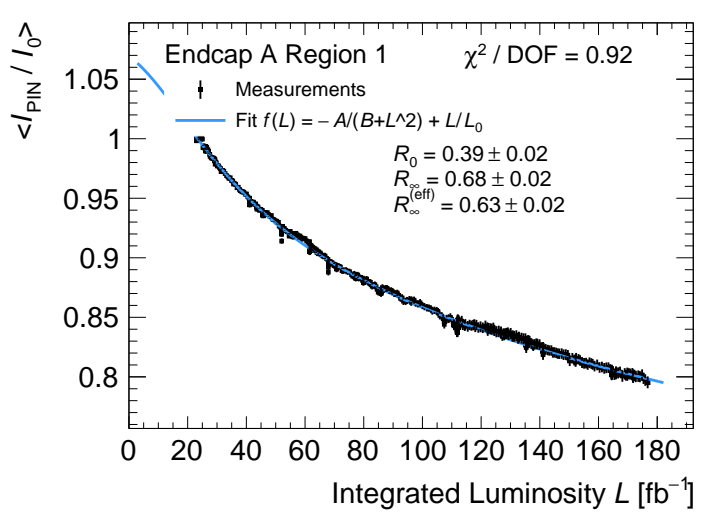

(a) End-cap A region 1.

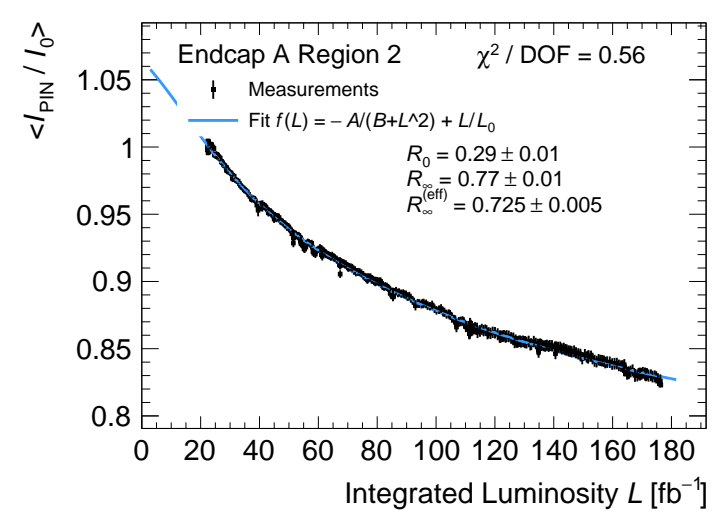

(b) End-cap A region 2.

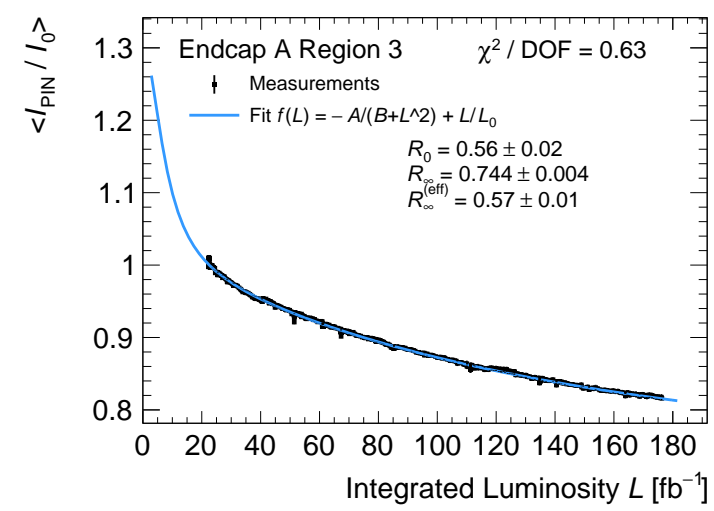

(c) End-cap A region 3.

Figure 13: End-cap A 2016, 2017 and 2018. The $\left\langle I_{\mathrm{PIN}} / I_{0}\right\rangle$ values of each region for End-cap A correlated against integrated luminosity (see text for details). The data is offset on the horizontal axis to allow for the effective luminosity delivered before 2016. Each point represents the mean value for one day averaged over all modules for each region. The value of $\left\langle I_{\mathrm{PIN}} / I_{0}\right\rangle$ is the normalised mean value of $I_{\text {PIN }}$ (see text for details). The curves show results of fits to the measurements using the parametrisation labelled in each plot. 


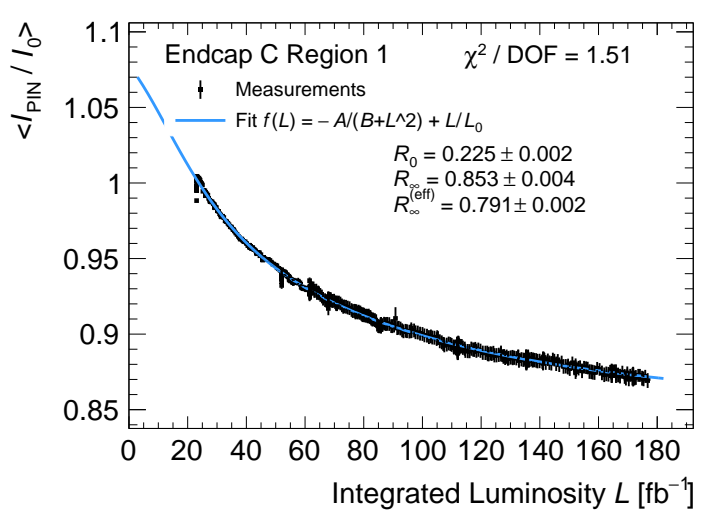

(a) End-cap C region 1.

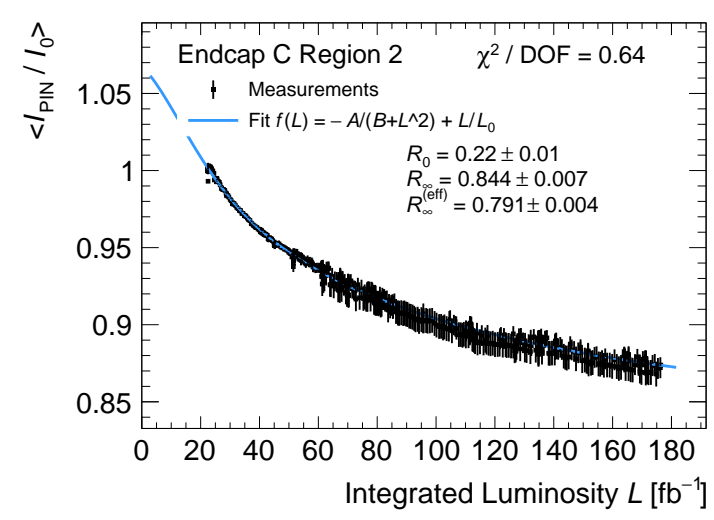

(b) End-cap C region 2.

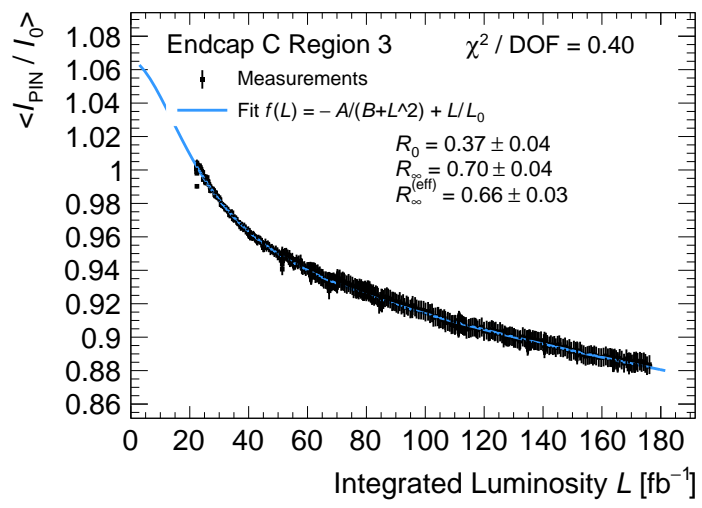

(c) End-cap C region 3.

Figure 14: End-cap C 2016, 2017 and 2018. The $\left\langle I_{\mathrm{PIN}} / I_{0}\right\rangle$ values of each region for End-cap C correlated against integrated luminosity (see text for details). The data is offset on the horizontal axis to allow for the effective luminosity delivered before 2016. Each point represents the mean value for one day averaged over all modules for each region. The value of $\left\langle I_{\mathrm{PIN}} / I_{0}\right\rangle$ is the normalised mean value of $I_{\text {PIN }}$ (see text for details). The curves show results of fits to the measurements using the parametrisation labelled in each plot. 


\section{Conclusion}

The results of the in situ radiation damage for the on-detector $p-i-n$ diodes and VCSELs used in the SCT optical links showed that these links should survive the radiation damage from the full LHC run. The VCSELs showed evidence for radiation damage as expected from test beam studies. The observed damage is larger than the extrapolation from test beam data using NIEL scaling in GaAs and this probably reflects the uncertainties in the NIEL scaling hypothesis for GaAs. The on-detector $p-i-n$ diodes showed clear evidence of radiation damage to the responsivity but no significant increase in depletion voltage. The effects of radiation damage up to the end of SCT operation on the VCSELs and $p-i-n$ diodes can be accommodated within the links power budget.

\section{A Stability study}

The stability of the measurements for the on-detector $p-i-n$ diodes was studied during periods in which there was no beam in the LHC. Three periods were considered: one in 2016, one in 2017 and one in 2018. In each case this consisted of the period up to the start of beam operation for which the temperature readings were stable. The stability was measured by plotting normalised values of the $p-i-n$ diode current as a function of time. The currents were normalised to the same values used for the analysis of the 2016, 2017 and 2018 data. The small variation in these normalised currents is probably due to fluctuations in the VCSEL fibre coupled power ${ }^{11}$. Therefore the results are shown for the 8 different readout ('ROD') crates which contain the VCSELs used to send light to the on-detector $p-i-n$ diodes. The results for the 2016, 2017 and 2018 data are shown in fig. 15, fig. 16 and fig. 17.

${ }^{11}$ The fluctuations in the VCSEL fibre coupled power are caused by (random) mode hopping of the VCSELs. VCSELs have one longitudinal mode but several transverse modes. Therefore mode hoping does not change the total power but as it changes the angles of the emitted light, it can have a large effect on the fibre coupled power. 


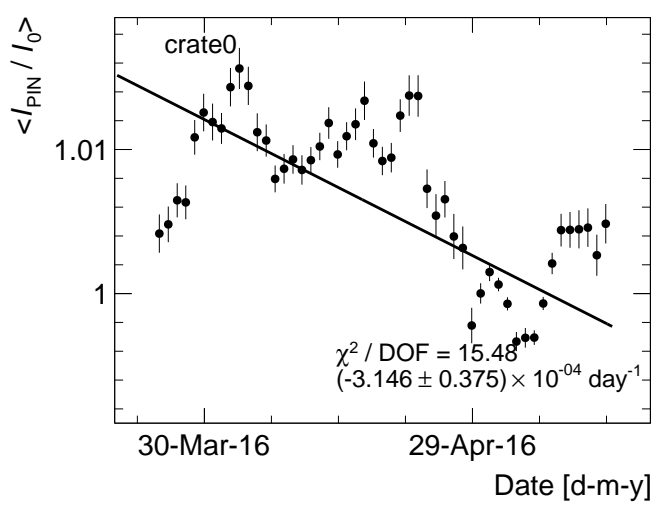

(a) Crate 0 .

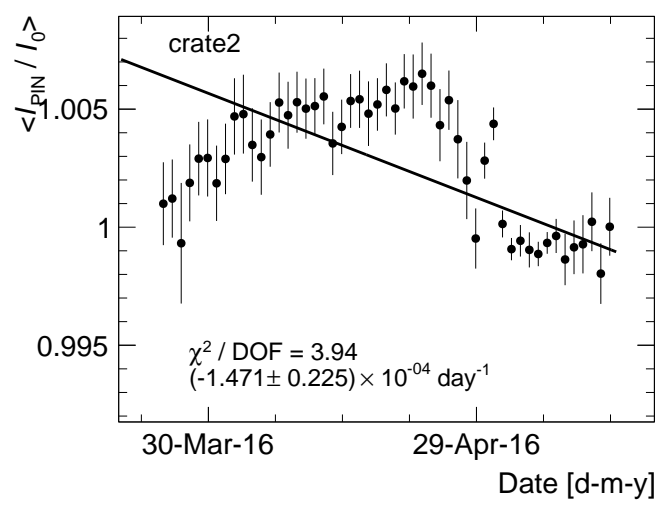

(c) Crate 2.

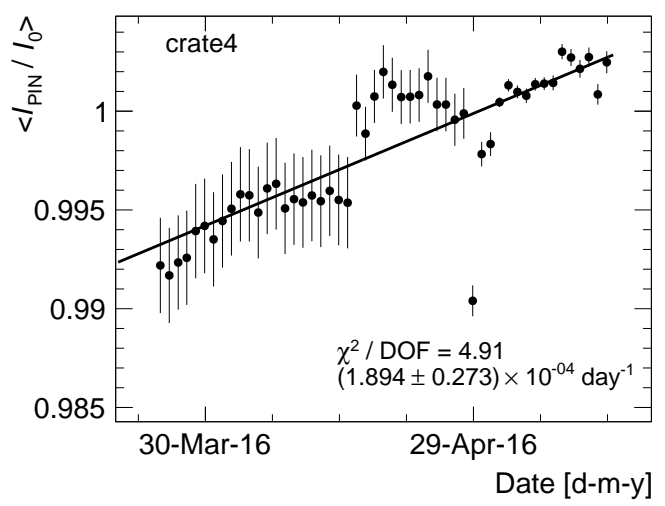

(e) Crate 4 .

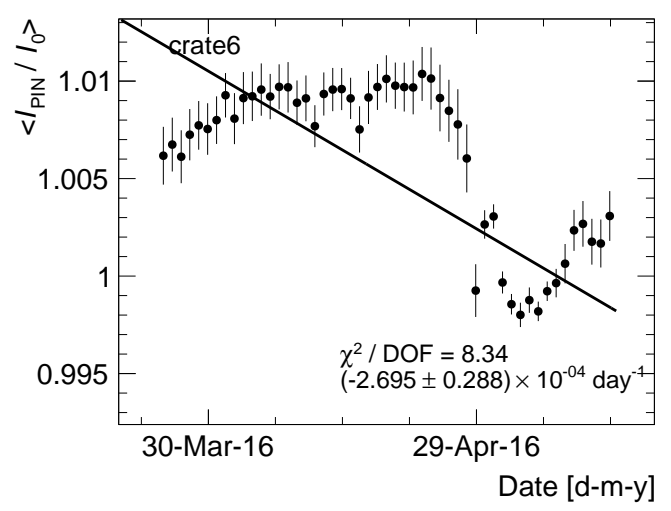

(g) Crate 6

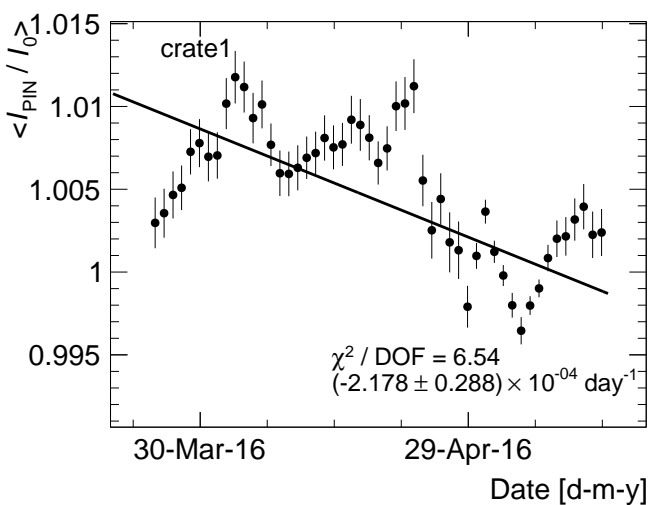

(b) Crate 1 .

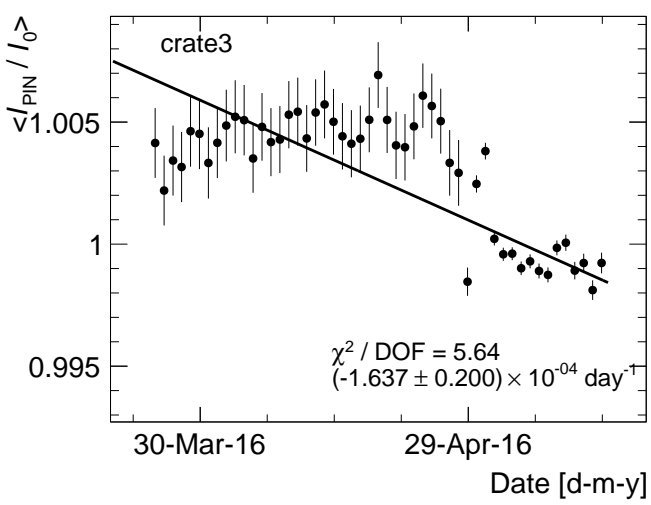

(d) Crate 3.

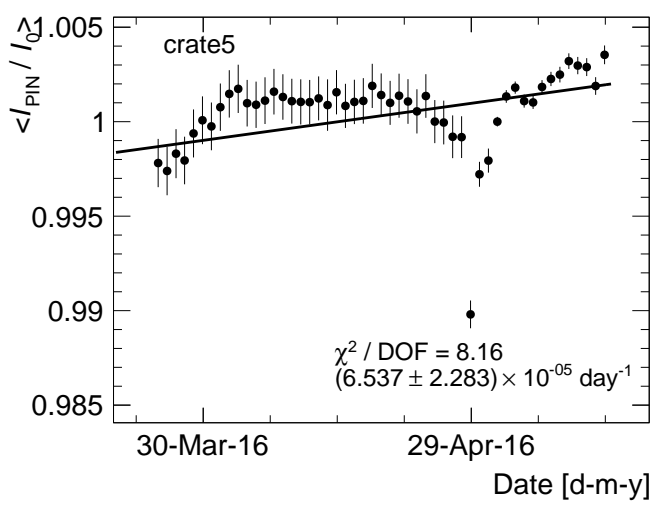

(f) Crate 5.

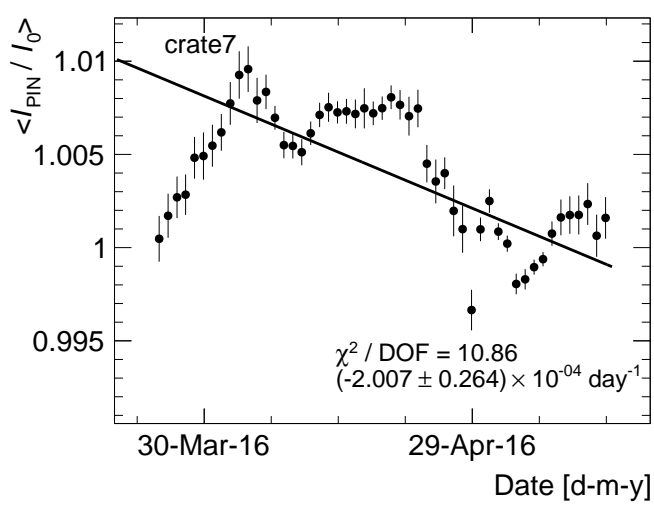

(h) Crate 7.

Figure 15: 2016. The $\left\langle I_{\mathrm{PIN}} / I_{0}\right\rangle$ values of each $\operatorname{R}_{2} \mathrm{OD}$ crate. Each point represents the mean value for one day averaged over all modules in a crate for the period before beam operation in 2016. The value of $\left\langle I_{\mathrm{PIN}} / I_{0}\right\rangle$ is the normalised mean value of $I_{\mathrm{PIN}}$ (see text for details). The line shows results of linear fits to the measurements. Note the highly suppressed zero on the vertical axis. 


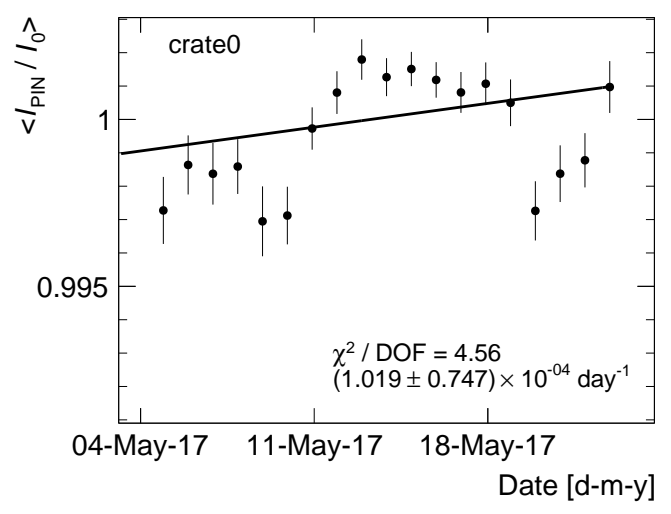

(a) Crate 0 .

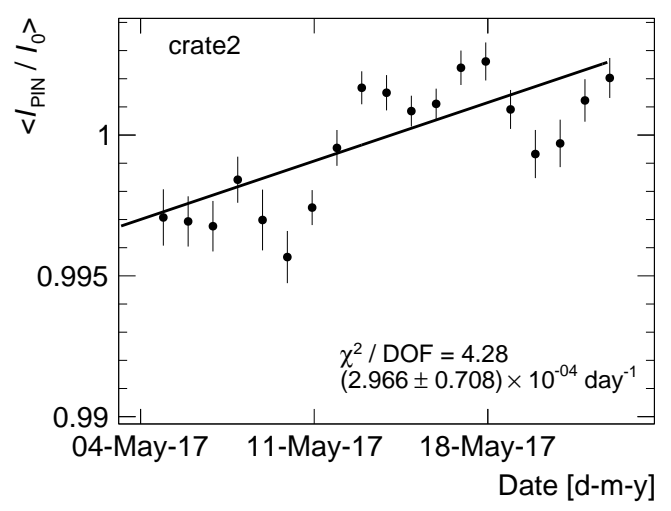

(c) Crate 2 .

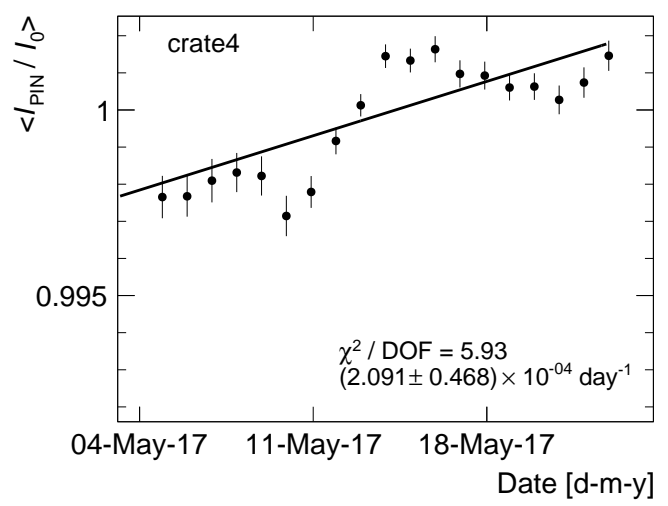

(e) Crate 4 .

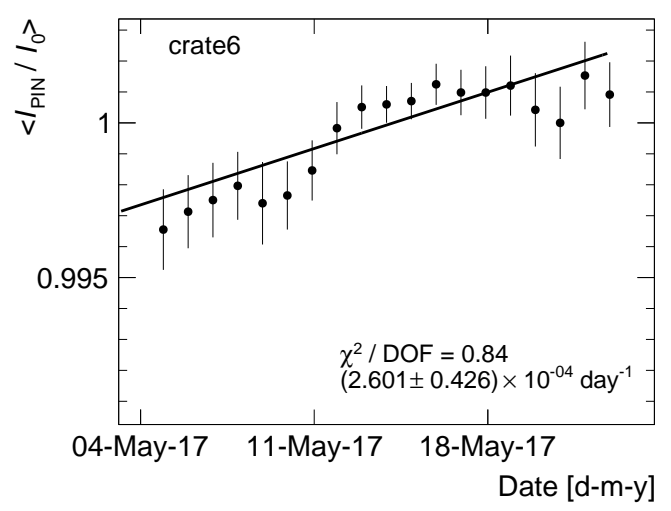

(g) Crate 6

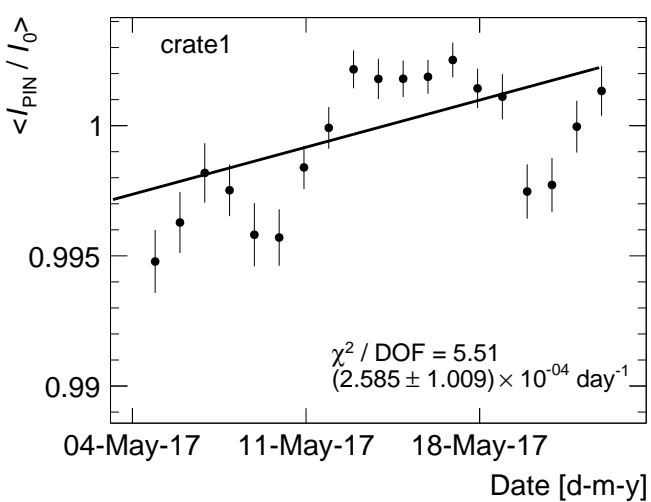

(b) Crate 1 .

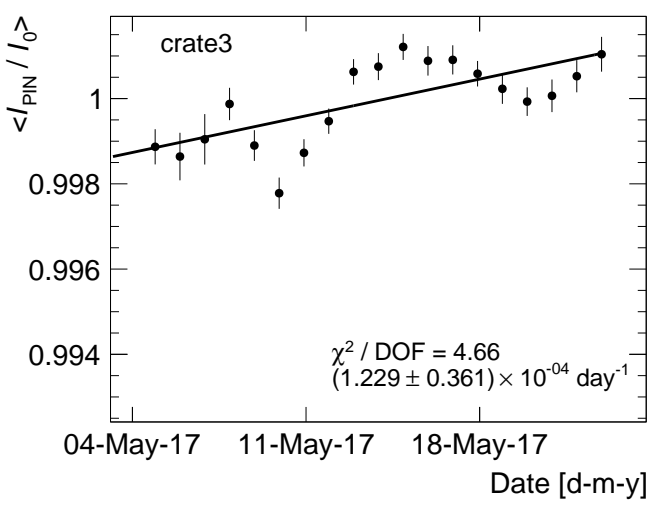

(d) Crate 3.

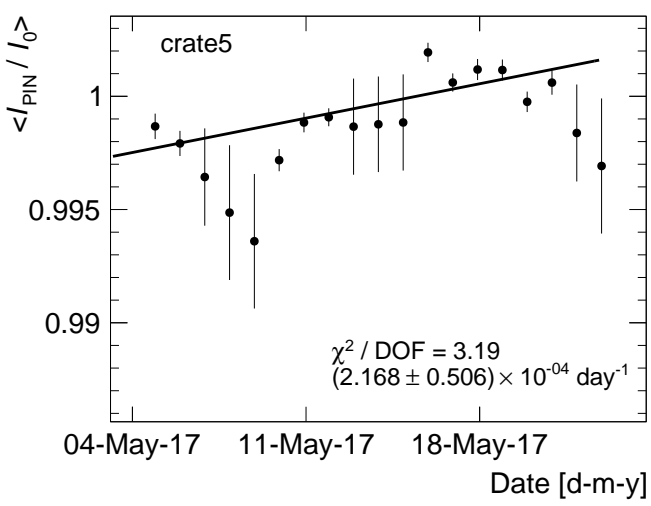

(f) Crate 5.

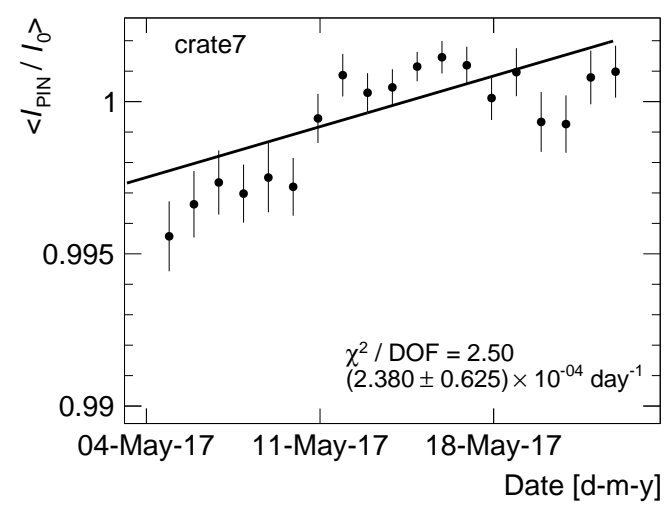

(h) Crate 7.

Figure 16: 2017. The $\left\langle I_{\mathrm{PIN}} / I_{0}\right\rangle$ values of each $28 \mathrm{OD}$ crate. Each point represents the mean value for one day averaged over all modules in a crate for the period before beam operation in 2017. The value of $\left\langle I_{\mathrm{PIN}} / I_{0}\right\rangle$ is the normalised mean value of $I_{\mathrm{PIN}}$ (see text for details). The line shows results of linear fits to the measurements. Note the highly suppressed zero on the vertical axis. 


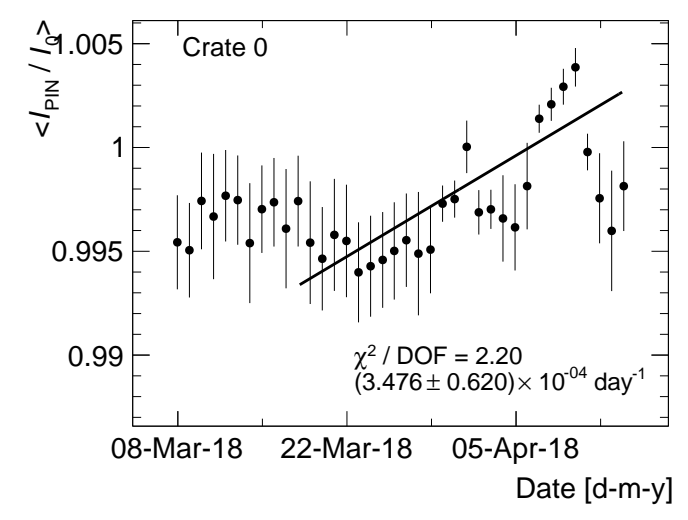

(a) Crate 0 .

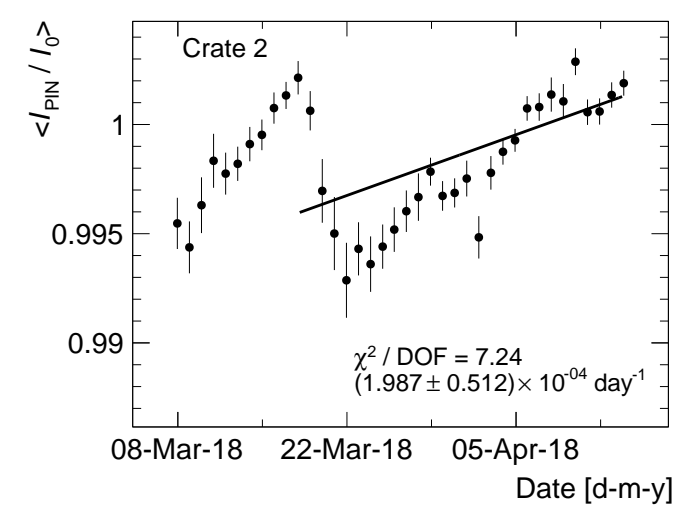

(c) Crate 2 .

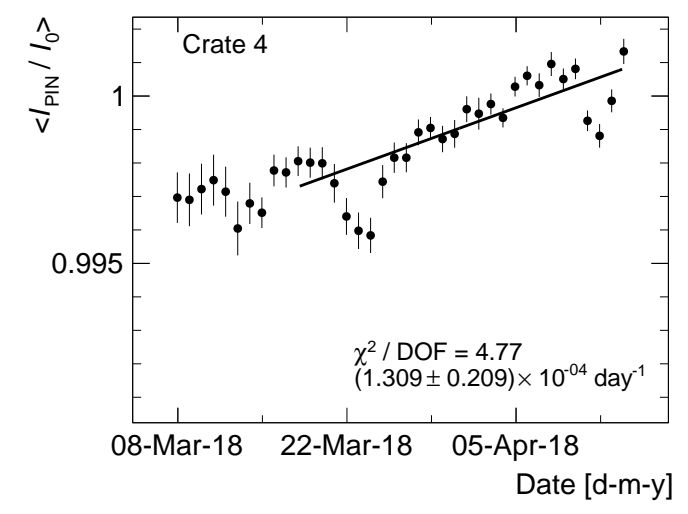

(e) Crate 4 .

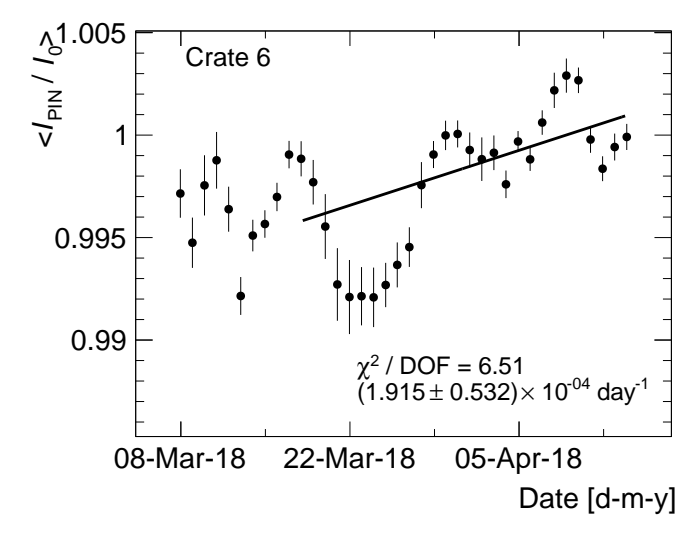

(g) Crate 6.

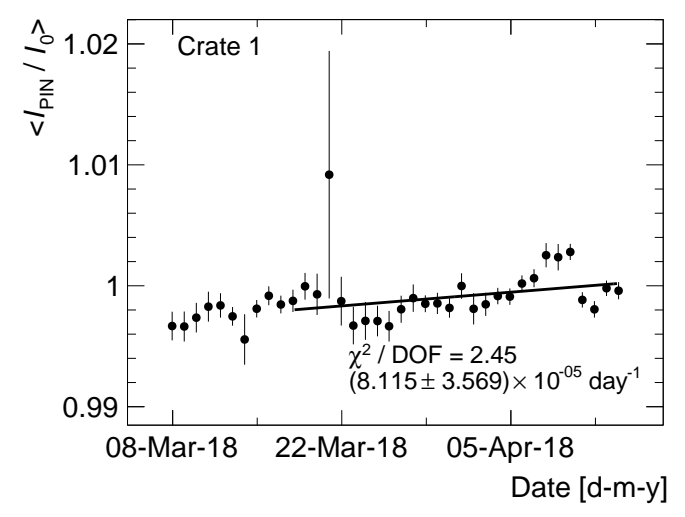

(b) Crate 1 .

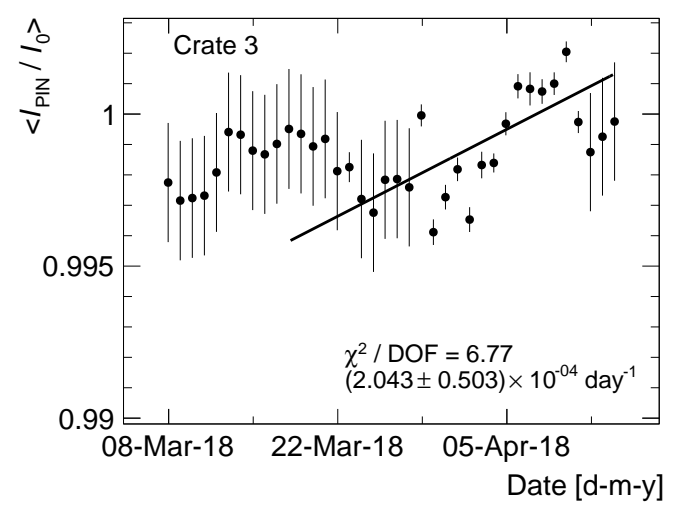

(d) Crate 3.

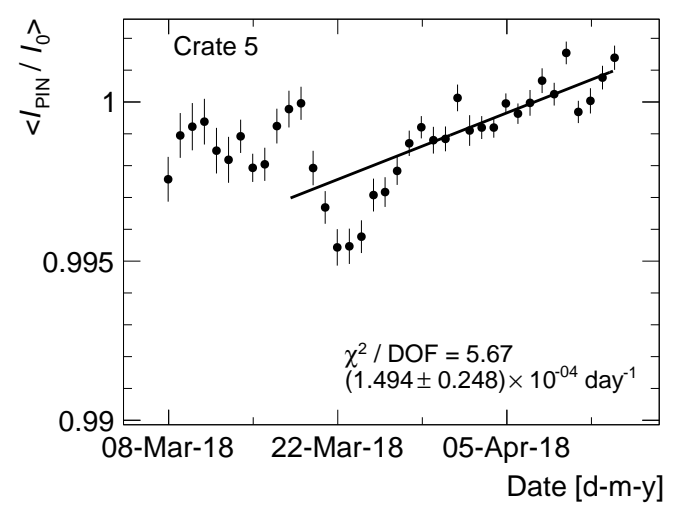

(f) Crate 5 .

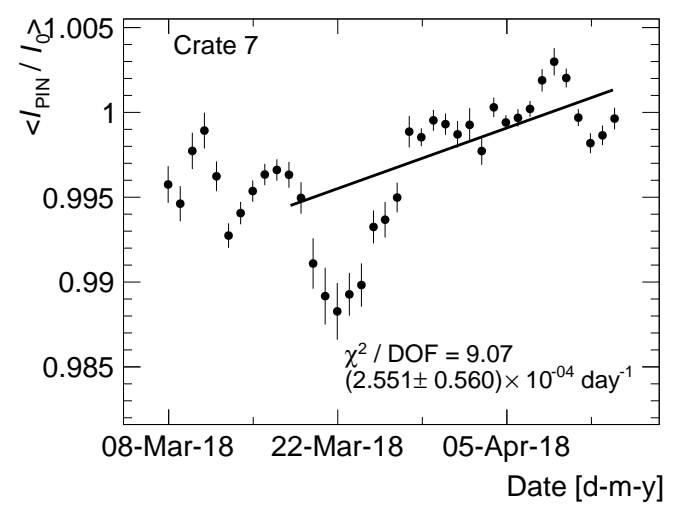

(h) Crate 7.

Figure 17: 2018. The $\left\langle I_{\mathrm{PIN}} / I_{0}\right\rangle$ values of each ${ }_{2} \rho-\rho_{-}$crate. Each point represents the mean value for one day averaged over all modules in a crate for the period before beam operation in 2018. The value of $\left\langle I_{\mathrm{PIN}} / I_{0}\right\rangle$ is the normalised mean value of $I_{\mathrm{PIN}}$ (see text for details). The line shows results of linear fits to the measurements. Note the highly suppressed zero on the vertical axis. 


\section{Acknowledgments}

Financial support from the Science and Technology Facilities Council (STFC, U.K.) is acknowledged. We thank the ATLAS collaboration for access to the data used in this paper. We also thank Dr. Igor Mandić (Jožef Stefan Institute and Department of Physics, University of Ljubljana) for useful discussions about the effects of radiation damage on $p-i-n$ diodes.

\section{References}

[1] ATLAS Collaboration. The ATLAS Experiment at the CERN Large Hadron Collider. J. Inst., 3: S08003, 2008. URL doi : 10.1088/1748-0221/3/08/S08003.

[2] Abdesselam, A. et. al. The optical links of the ATLAS SemiConductor tracker. JINST, 2:P09003, 2007. URL http://iopscience.iop.org/1748-0221/2/09/P09003.

[3] The ATLAS collaboration. Operation and performance of the atlas semiconductor tracker. J. Inst., 9 (08):P08009, 2014. URL http://stacks .iop.org/1748-0221/9/i=08/a=P08009.

[4] ATLAS Collaboration. Luminosity determination in pp collisions at $\sqrt{s}=8 \mathrm{TeV}$ using the ATLAS detector at the LHC. Eur. Phys. J., C 76:653, 2016. URL arXiv: 1608.03953.

[5] Lindstrom, G. Moll, M. and Fretwust, E. Radiation hardness of silicon detectors, a challenge from high-energy physics. Nucl. Instr. Meth., A426:1, 1999. URL https://doi .org/10.1016/S0168-9002(98)01462-4.

[6] Summers, G et. al. DAMAGE CORRELATIONS IN SEMICONDUCTORS EXPOSED TO GAMMA, ELECTRON AND PROTON RADIATIONS. IEEE TRANSACTIONS ON NUCLEAR SCIENCE, 40 NO. 6:1372, 1993. doi: 10.1109/23.273529. URL http://dx. doi .org/10.1109/23.273529.

[7] Beringer, J. et. al. Radiation hardness and lifetime studies of LEDs and VCSELs for the optical readout of the ATLAS SCT. Nucl. Instr. Meth., A435:375, 1999. URL https://doi .org/10.1016/S0168-9002(99)00570-7.

[8] Teng, P. K. et. al. Radiation hardness and lifetime studies of the VCSELs for the ATLAS semiconductor tracker. Nucl. Instr. Meth., A497:294, 2003. URL https://doi .org/10.1016/S0168-9002(02)01922-8.

[9] Stejskal P. et. al. Modelling radiation-effects and annealing in semiconductor lasers for use in future particle physics experiments. J. Inst., 6:C12045, 2011. URL http://dx.doi.org/10.1088/1748-0221/6/12/C12045.

[10] Dowell, J.D. et. al. Irradiation test of photodiodes for the ATLAS SCT readout. Nucl. Instr. Meth., A424:483, 1999. URL https://doi.org/10.1016/S0168-9002(98)01415-6.

[11] Charlton, D.G. et. al. Radiation hardness and lifetime studies of photodiodes for the optical readout of the ATLAS semiconducotr tracker. Nucl. Instr. Meth., A456:300, 2001. URL https://doi .org/10.1016/S0168-9002(00)00666-5.

[12] Ferrari A, Sala P.R, Fasso A. and Ranft J. FLUKA: A multi-particle transport code (Program version 2005), CERN-2005-010, SLAC-R-773, INFN-TC-05-11. http://cdsweb. cern.ch/record/898301.

[13] Battistoni G. et. al. The FLUKA code: description and benchmarking. AIP Conf. Proc. 896 (2007) 31. 
[14] Baranov S., Bosman M., Dawson I., Hedberg V., Nisati A. and Shupe M. Estimation of Radiation Background, Impact on Detectors, Activation and Shielding Optimization in ATLAS, ATL-GEN-2005-001. URL http: //cdsweb . cern. ch/record/814823.

[15] Sjöstrand T., Mrenna S., and Skands P.Z. A brief introduction to PYTHIA 8.1. Comput. Phys. Commun., 178:852, 2008. URL arXiv:0710. 3820.

[16] ATLAS Collaboration. A study of the Pythia 8 description of ATLAS minimum bias measurements with the Donnachie-Landshoff diffractive model, ATL-PHYS-PUB-2016-017. https: //cds . cern. ch/record/2206965, 2016.

[17] ATLAS Collaboration. ATLAS Inner Tracker Strip Detector, Technical Design Report, ATL-TDR-025, CERN LHCC-2017-005. https : //cds . cern. ch/record/2257755, 2017.

[18] Chu, M-L et. al. The Off-Detector Opto-electronics for the Optical Links of the ATLAS SemiConductor Tracker and Pixel Detector. Nucl. Instr. Meth., A530:293, 2004. URL https://doi.org/10.1016/j.nima.2004.04.228.

[19] Weidberg, A. VCSEL reliability in ATLAS and development of robust arrays. J. Inst., 7:C01098, 2012. URL http://dx . doi .org/10.1088/1748-0221/7/01/C01098.

[20] Helmuth Spieler. Semiconductor Detector Systems. Oxford University Press, 2005. 